\title{
Algorithm for theoretical mapping of bio-strings for co-expression: bridging genotype to phenotype
}

\author{
Om Prakash \\ Department of Computational Biology \\ The Institute of Mathematical Sciences, Chennai-600113 \\ Email: mailbox4op@gmail.com
}

\begin{abstract}
Identification of possibility of co-expression or co-performance directly from set of bio-strings as protein sequences represents an important problem of bridging between genotype to phenotype. This algorithm presents above bridging. Algorithm was implemented with proteins known from human hormone signaling system. Co-expression of proteins was cross-validated through human gene COXPRESdb v7 database. Possibility of protein-protein interaction (PPI) was also cross checked through STRING database. Results were found to be effectively fascinating. Considering the indications from results, this algorithm can be adopted for theoretical identification of co-expression or co-performance of bio-strings.
\end{abstract}

Keywords: Algorithm; Co-expression; Map; Polarized; Sequence

\section{INTRODUCTION}

Relating genotype to phenotype by co-expression of objects in a system bears an enthusiastic attention in biological research. Co-expression is well defined through multiple empirical ways. There are evidences describing relation between genotype and phenotypes at different strata of studies. As in a study co-expression of protein variants and inter-allelic effects were observed as genotype-phenotype (Shen et al., 2016). Integration was established among diet, APOE genotype and immune response (Nam et al., 2018). Host genotype and tumor phenotype was also mentioned to be mapped in case of breast cancer (Yu et al., 2015). Structural and functional aspects were also understood as genotype-phenotype correlations (Shen et al., 2016), which supported the relationships in the light of natural selection (Chen et al., 2018). Gene modules and salt stress in rice were analyzed through network mapping (Du et al., 
2019). Weighted gene co-expression was analyzed through network mapping (Eidsaa et al., 2017). Genetic mutation in CLCNKB was correlated with phenotypes in patients (Cheng et al., 2017). Beyond these, effect of de novo mutations in YWHAG was observed for Early-Onset Epilepsy (Guella et al., 2017). Functional modules were searched for Co-expression (Schaefer et al., 2014). Further Insights were drawn in the most common SCN5A mutation causing overlapping phenotype of long QT syndrome, Brugada syndrome, and conduction defect (Veltmann et al., 2016). Genomic and transcriptomic information were integrated for identification of genetic markers (Jung et al., 2019). Beyond these, no theoretical aspect defined till now for co-expression for bio-strings. Theoretical description about coexpression may unravel multiple aspects of co-expression other than empirical evidences.

Present algorithm presents bridging between geno \& pheno types. Since, Time is the scaling factor for co-expression of two objects; therefore system objects will be known to be co-expressed if they are present at same instance of time. This factor is independent of constraint of location for expression, although multiple factors participate during expression of different factors at different locations. In present study, the theoretical seed base has been presented for bridging between biostring-pairs (here protein sequence) and their possible co-expression. Algorithm was implemented with proteins known for human hormone signaling system. The algorithm presented a generalized base for observation of bio-string pairs in reference of their possible co-expression.

\section{MATERIAL AND METHOD}

Dataset for mapping: Protein set was collected from NCBI. Set included known protein sequences (and respective UniProt IDs) from hormone signaling systems involved in multiple diseases of human. The set included 33 unique protein objects.

Pseudo-code of Algorithm: Initial requirement for starting the algorithm was a set of bio-strings (here protein sequence), each string out of set was transformed into 'sequence memory map' and further processed for identification of co-expression bio-string pairs. Whole algorithm has been divided into 04 major steps with pseudo codes.

\section{Step1: Developing Sequence Memory Map (SMM)}

Firstly initialize the SMM as zero matrix of order $u \times w$. In fact $u$ is the number of unique characters in the data type (as for nucleotide sequence $u=4$, and for protein sequence $u=20$ ). Number of columns w represented window-length used for map enrichment. 
Forward enrichment of SMM: Enrich the zero matrix with elemental observation of string. Each element of string throws a value into matrix in respect of their position in string as well as unique character. Memory matrix has been filled with respective value of string element.

Let $\mathrm{O}_{\mathrm{uxv}}$ be a SMM zero matrix of order $\mathrm{u} \times \mathrm{v}$. Let $\mathrm{S}=\left\{\mathrm{s}_{\mathrm{n}}\right\}$ be a string of length $\mathrm{n}(\mathrm{s})$. Let Let $U=$ $\langle u 1, u 2, u 3, u 4, u 5, u 6, u 7, u 8, u 9, u 10, u 11, u 12, u 13, u 14, u 15, u 16, u 17, u 18, u 19, u 20\rangle \quad$ an ordered set, where $u 1, u 2, u 3, u 4, u 5, u 6, u 7, u 8, u 9, u 10, u 11, u 12, u 13, u 14, u 15, u 16, u 17, u 18, u 19, u 20$ are denoted by $A, C, D, E, F, G, H, I, K, L, M, N, P, Q, R, S, T, V, W, Y$ respectively. (If nucleotide sequences were consider, then $\mathrm{U}=\langle u 1, u 2, u 3, u 4>$ for $\mathrm{A}, \mathrm{T}, \mathrm{G}, \mathrm{C}$ respectively). Here $\mathrm{w}$ denotes window size. Let $d=\lceil n(s) / w\rceil$ the smallest integer $\geq n(s) / w$. Let $\mathrm{W}_{\mathrm{nc}}$ denote the rearranged string $\mathrm{S}$ in the matrix of order $\mathrm{d} \times \mathrm{c}$ (Blank element will be considered, if required during rearrangement).

$$
\left[\left[\left(\left(W_{n c}\right)_{c=1}^{w}\right) \rightarrow\left(U_{k}\right)_{k=1}^{n(u)}\right] \Rightarrow\left(o_{u v}=1+\sum_{x=1}^{n(u)} o_{x v}\right)_{v=1}^{w}\right]_{n=1}^{d}
$$

Back-tracing the sequence element (SE) from SMM map: Backward tracing has been adapted. $U_{u}$ is the character of string back-traced from SMM. SMM should be updated after each cycle of back-trace.

$$
\begin{gathered}
{\left[\left(\left(\left(n(U) *(i)_{i=1}^{n(U)}\right)+(j)_{j=1}^{v}\right) \rightarrow(z)_{z=1}^{v}\right) \wedge z\right] \Rightarrow v} \\
{\left[\left\{\left(\boldsymbol{O}_{\boldsymbol{u} v}\right)_{\boldsymbol{u}=1}^{n(U)}\right\}_{\text {max }}\right] \Rightarrow \mathrm{u}}
\end{gathered}
$$

Back traced String element $=U_{u}$

\section{Step2: Generating string pair combinations with different window size}

By calculating Euclidean distance between two SMM maps, each string pair has been represented by SMM distance value. Matrix of order $n \times m$ was filled with element wise Euclidian distance been two SMMs.

$$
[n \times m]=\text { euclidian }\left((S M M)_{1} \sim(S M M)_{2}\right)
$$

\section{Step3: Estimation of orthogonal components}

Orthogonal components were estimated by Principal Component Analysis method

$$
(p 1, p 2)=p c a([n \times m])
$$

\section{Step4: Defining Time Equivalent Factor (TEF) for co-expression}

By implementing Polarization on pattern \& clustering of co-expressed protein pairs, Coexpressed pairs were identified as TEF approaching zero. TEF has been defined as:

$$
[(T E F:=|\operatorname{atan}(p 1 / p 2) \sim \operatorname{atan}(p 2 / p 1)|) \rightarrow(\approx \text { zero })] \Rightarrow \text { Coexpressed }
$$




\section{Experimental evaluation of Algorithm:}

Algorithm was evaluated by mapping a set of 33 protein hormones filtered (from NCBI database) for human against multiple diseases. The protein sequences were collected with IDs and other details. Each sequence was processed through algorithm described above, memorized into SMM matrix and all paired combinations of proteins were marked on the basis of time equivalent factor (TEF). TEF $<=0.1$ has been tabulated and cross checked through COXPRESdb v7 database. The resulting proteins were also cross checked for their possibilities of mutual interaction through STRING database.

\section{RESULTS \& DISCUSSION}

Co-expression or co-occurrence of biomolecules is an evidence of existence at same time instance or in range of small time. Therefore for theoretical identification of co-expression, it became important to define scaling factor equivalent to Time. So that biomolecules could be evaluated on the ground of coexpression. This time equivalent factor (TEF) worked as independent from constraint of location for expression. The algorithm presented a generalized base for processing of protein pairs for defining coexpression. Algorithm took input of string out of a set of protein sequences and transformed into 'sequence memory map', which was further processed for calculation of TEF for each protein pairs. The algorithm has been described in 04 parts. Sequence Memory Map (SMM) was a matrix of order $20 \times \mathrm{w}$, where 20 represented twenty amino acids in a fixed order, and $w$ represented window size of sub-string used for enrichment of SMM. Algorithm has been processed in such a way that the mapped string can be back-traced from SMM itself. To generate pattern from SMM, distance between proteins of pair was tabulated by using ten different window sizes of 06 to 15 . The tabulated data was processed for calculation of orthogonal components. First two components were used for defining TEF by polarization of data. Finally TEF was used for filtering protein-pairs as co-expressed biomolecules. Algorithm was programmatically written as Java script and implemented with combinations of hormone proteins involved in human diseases. For experimental evaluation, TEF $<=0.1$ was used for picking up protein pairs approaching zero. By cross checking the theoretically identified results through COXPRESdb v7 and STRING databases, fascinating results were observed.

Raw data prepared from ten different windows was processed for analysis. Protein-pair combinations were clustered on the frame of first two orthogonal components. Data found to be arranged in some specific global pattern along with local angular discrete point arrangement. Angular arrangement flow 
provided indicatives towards the polarizing tendency into paired data structure (Figure 1). Polarity observations on orthogonal frame were plotted separately. Both Unidirectional \& bidirectional discrete behavior was observed (Figure 2). Regression of polarized vectors spreads the data points into two classes of opposite poles. Absolute values of difference in polar spread in an equivalent indicative of time therefore were used as factor of selecting co-expression. Data points approaching zero have been considered as co-expressed. By processing the data polarization, discrete points on orthogonal frame were grouped into two clusters (Figure 3). Experimental evaluation of results obtained by theoretically defined co-expression via algorithm. Good extents of experimental evidences were observed out of theoretically defined co-expressed protein-pairs. Polarization segregated the datasets into two groups with different protein pairs. TEF $<=0.1$ based filtering provided most possible pairs. Out of the TEF filtered results, experimentally known pairs were remarked as 'EA'. (EA: Experimentally Approved (through COXPRESdb v7 Database); P: Predicted possibility) (Table 1). STRING database was also queried with following list for observation of PPI along with co-expression (Table 2). Protein-protein interaction networks by using STRING database, showing possible protein-protein interactions observed among the theoretically defined co-expression of protein pairs. BLACK linking presents co-expression, MAGENTA linking presents experimentally determined link, Light GREEN presents text-mining based interaction possibility and BLUE links present gene co-occurrence (Figure 4). By combined interpretation of results observed through two databases COXPRESdb and STRING, it was observed that STRING did not showed any interaction for GHSR (growth hormone secretagogue receptor), THRB (thyroid hormone receptor beta) and RNPC3 (RNA binding region (RNP1, RRM) containing 3). But COXPRESdb showed that GHSR is co-expressed with LHX3 (LIM homeobox 3), THRB is co-expressed with MC2R (melanocortin 2 receptor) \& POU1F1 (POU class 1 homeobox 1), and RNPC3 is co-expressed with GNAS (GNAS complex locus). These co-expression results were also shown by present algorithm. Conclusively, the predicted coexpressions have high chances to be experimentally proved in future experiments. 


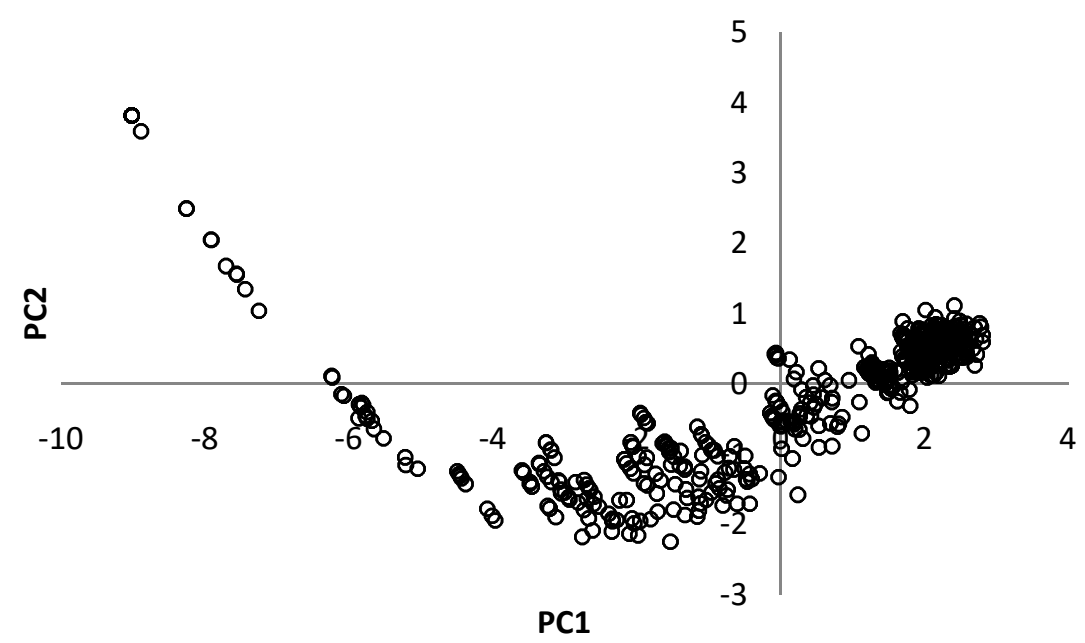

Figure 1. Protein-pair combinations were clustered on the frame of first two orthogonal components. Data found to be arranged in some specific global pattern along with local angular discrete point arrangement. Angular arrangement flow provided indicatives towards the polarizing tendency into paired data structure.
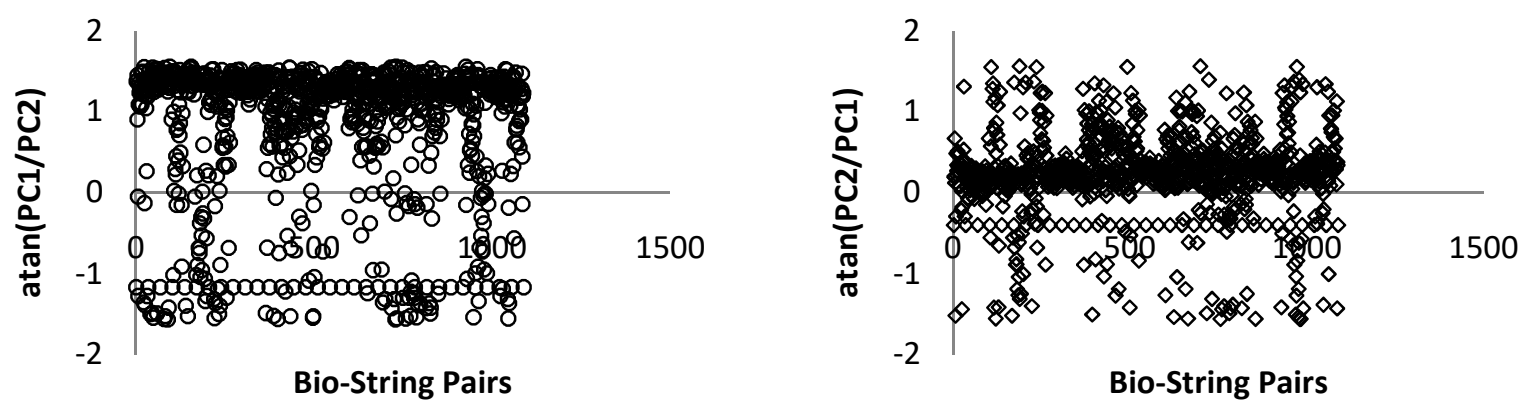

Figure 2. Polarity observations on orthogonal frame were plotted separately. Both Unidirectional \& bidirectional discrete behavior was observed. 


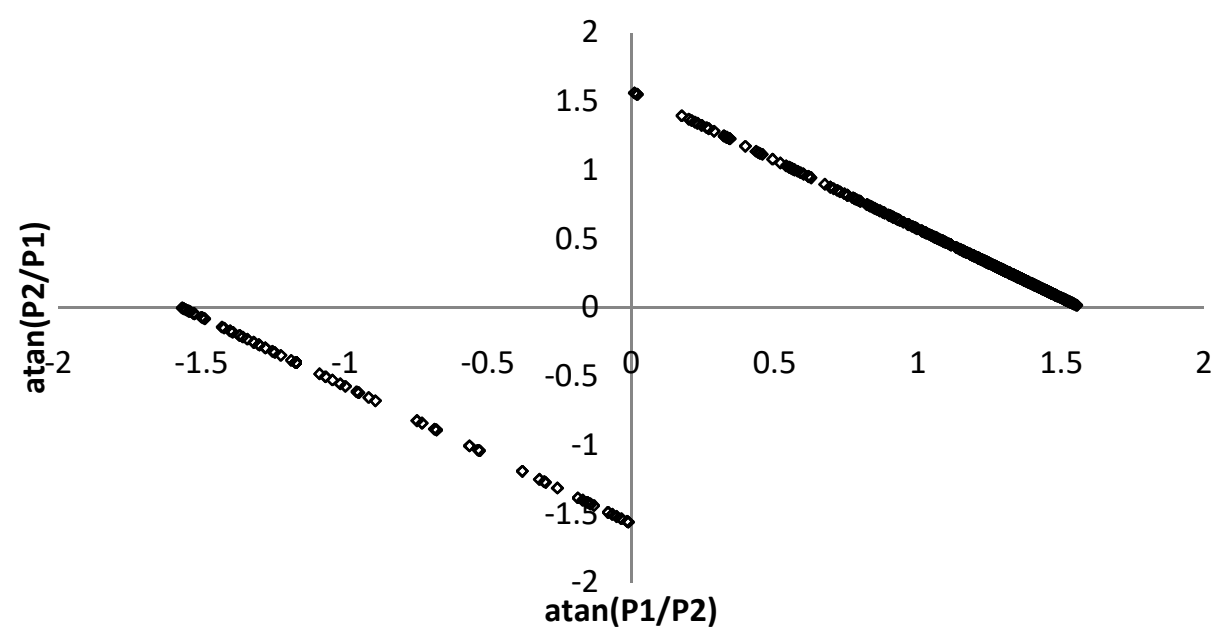

Figure 3. Regression of polarized vectors spreads the data points into two classes of opposite poles. Absolute values of difference in polar spread in an equivalent indicative of time therefore were used as factor of selecting co-expression. Data points approaching zero have been considered as co-expressed. By processing the data polarization, discrete points on orthogonal frame were grouped into two clusters.

Table 1. Experimental evaluation of results obtained by theoretically defined co-expression via algorithm. Good extents of experimental evidences were observed out of theoretically defined coexpressed protein-pairs. Polarization segregated the datasets into two groups with different protein pairs. TEF $<=0.1$ based filtering provided most possible pairs. Out of the TEF filtered results, experimentally known pairs were remarked as 'EA'. (EA: Experimentally Approved (through COXPRESdb v7 Database); P: Predicted possibility).

\begin{tabular}{|c|c|c|c|c|c|c|}
\hline $\begin{array}{c}\text { Cluster } \\
\text { no. }\end{array}$ & Protein 1 & Protein 2 & $\begin{array}{c}\text { Entrez } \\
\text { Gene ID } \\
\text { (Protein 1) }\end{array}$ & $\begin{array}{c}\text { Entrez } \\
\text { Gene ID } \\
\text { (Protein 2) }\end{array}$ & TEF & Remarks \\
\hline \multirow{4}{*}{ Group-1 } & Q96LT9 & P63092 & $\mathbf{5 5 5 9 9}$ & $\mathbf{2 7 7 8}$ & $\mathbf{0 . 0 7 4 8 4 1}$ & EA \\
\cline { 2 - 7 } & P63092 & Q96LT9 & 2778 & 55599 & 0.074841 & P \\
\hline \multirow{5}{*}{ Group-2 } & P10828 & Q01718 & $\mathbf{7 0 6 8}$ & $\mathbf{4 1 5 8}$ & $\mathbf{0 . 0 2 2 7 3 5}$ & EA \\
\cline { 2 - 7 } & P10828 & P28069 & $\mathbf{7 0 6 8}$ & $\mathbf{5 4 4 9}$ & $\mathbf{0 . 0 1 9 7 0 7}$ & EA \\
\cline { 2 - 7 } & Q969G2 & P10644 & $\mathbf{8 9 8 8 4}$ & $\mathbf{5 5 7 3}$ & $\mathbf{0 . 0 2 8 5 6}$ & EA \\
\cline { 2 - 7 } & Q969G2 & Q92847 & $\mathbf{8 9 8 8 4}$ & $\mathbf{2 6 9 3}$ & $\mathbf{0 . 0 1 2 8 8 9}$ & EA \\
\cline { 2 - 7 } & P63092 & Q9UBR4 & 2778 & 8022 & 0.061385 & P \\
\cline { 2 - 7 } & P63092 & O00170 & 2778 & 9049 & 0.08347 & P \\
\cline { 2 - 7 } & P63092 & Q92847 & $\mathbf{2 7 7 8}$ & $\mathbf{2 6 9 3}$ & $\mathbf{0 . 0 0 2 6 8 3}$ & EA \\
\cline { 2 - 7 } & Q9UBR4 & P63092 & $\mathbf{8 0 2 2}$ & $\mathbf{2 7 7 8}$ & $\mathbf{0 . 0 6 1 3 8 5}$ & EA \\
\cline { 2 - 7 } & Q9UBR4 & Q06710 & $\mathbf{8 0 2 2}$ & $\mathbf{7 8 4 9}$ & $\mathbf{0 . 0 7 4 5 1 7}$ & EA \\
\cline { 2 - 7 } & Q9UBR4 & Q92847 & 8022 & 2693 & 0.076391 & P \\
\hline
\end{tabular}




\begin{tabular}{|c|c|c|c|c|c|}
\hline Q01718 & P10828 & 4158 & 7068 & 0.022735 & $P$ \\
\hline P41225 & 000170 & 6658 & 9049 & 0.027282 & $\mathrm{P}$ \\
\hline P28069 & P10828 & 5449 & 7068 & 0.019707 & $P$ \\
\hline P10644 & Q969G2 & 5573 & 89884 & 0.02856 & $P$ \\
\hline P10644 & P63092 & 5573 & 2778 & 0.060578 & EA \\
\hline Q06710 & Q9UBR4 & 7849 & 8022 & 0.074517 & $P$ \\
\hline P32243 & 060806 & 5015 & 9095 & 0.092159 & $\mathrm{P}$ \\
\hline 000170 & P63092 & 9049 & 2778 & 0.08347 & EA \\
\hline 000170 & P41225 & 9049 & 6658 & 0.027282 & EA \\
\hline 060806 & P32243 & 9095 & 5015 & 0.092159 & EA \\
\hline Q92847 & Q969G2 & 2693 & 89884 & 0.012889 & $P$ \\
\hline Q92847 & P63092 & 2693 & 2778 & 0.002683 & $\mathrm{P}$ \\
\hline Q92847 & Q9UBR4 & 2693 & 8022 & 0.076391 & EA \\
\hline
\end{tabular}

Table 2. STRING database was also queried with following list for observation of PPI along with coexpression

\begin{tabular}{|l|l|r|}
\hline Gene Symbol & \multicolumn{1}{|c|}{ Function } & Entrez Gene ID \\
\hline GHSR & growth hormone secretagogue receptor & 2693 \\
\hline GNAS & GNAS complex locus & 2778 \\
\hline MC2R & melanocortin 2 receptor & 4158 \\
\hline OTX2 & orthodenticle homeobox 2 & 5015 \\
\hline POU1F1 & POU class 1 homeobox 1 & 5449 \\
\hline PRKAR1A & protein kinase cAMP-dependent type I regulatory subunit alpha & 5573 \\
\hline SOX3 & SRY-box 3 & 6658 \\
\hline THRB & thyroid hormone receptor beta & 7068 \\
\hline PAX8 & paired box 8 & 7849 \\
\hline LHX3 & LIM homeobox 3 & 8022 \\
\hline AIP & aryl hydrocarbon receptor interacting protein & 9049 \\
\hline TBX19 & T-box 19 & 9095 \\
\hline RNPC3 & RNA binding region (RNP1, RRM) containing 3 & 55599 \\
\hline LHX4 & LIM homeobox 4 & 89884 \\
\hline
\end{tabular}




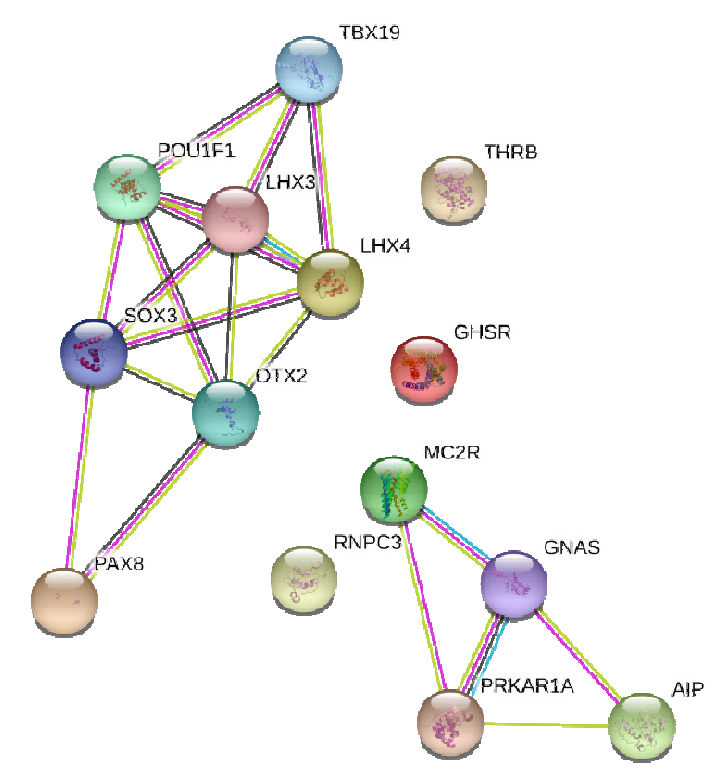

Figure 4. Protein-protein interaction networks by using STRING database, showing possible proteinprotein interactions observed among the theoretically defined co-expression of protein pairs. BLACK linking presents co-expression, MAGENTA linking presents experimentally determined link, Light GREEN presents text-mining based interaction possibility and BLUE links present gene co-occurrence.

\section{CONCLUSION}

Theoretical co-expression or co-performance can be indicated directly from set of bio-strings. Algorithm has been implemented for bridging between genotype to phenotype. Considering the results found, algorithm can be adopted for theoretical identification of co-expression or co-performance of biostrings.

\section{Supplementary Tables: S1 (List of protein hormones related with human diseases); and S2: (Protein} pairs evaluated for co-expression)

\section{ACKNOWLEDGEMENT}

Author express gratitude to The Institute of Mathematical Sciences, Chennai-600113, India for providing research facilities as well as DAE Post-Doctoral Fellowship (PDF 214). Author is also thankful to Dr. Amit Singh (PDF Mathematics, IMSc Chennai) for mathematical proof editing.

\section{REFERENCES}

Chen, H., Wu, C.I., He, X., 2018. The genotype-phenotype relationships in the light of natural selection. Mol. Biol. Evol. https://doi.org/10.1093/molbev/msx288 
Cheng, C.J., Lo, Y.F., Chen, J.C., Huang, C.L., Lin, S.H., 2017. Functional severity of CLCNKB mutations correlates with phenotypes in patients with classic Bartter's syndrome. J. Physiol. 595, 5573-5586. https://doi.org/10.1113/JP274344

Du, Q., Campbell, M., Yu, H., Liu, K., Walia, H., Zhang, Q., Zhang, C., 2019. Network-based feature selection reveals substructures of gene modules responding to salt stress in rice. Plant Direct. https://doi.org/10.1002/pld3.154

Eidsaa, M., Stubbs, L., Almaas, E., 2017. Comparative analysis of weighted gene coexpression networks in human and mouse. PLoS One 12. https://doi.org/10.1371/journal.pone.0187611

Guella, I., McKenzie, M.B., Evans, D.M., Buerki, S.E., Toyota, E.B., Van Allen, M.I., Adam, S., Boelman, C., Bolbocean, C., Candido, T., Eydoux, P., Horvath, G., Huh, L., Nelson, T.N., Sinclair, G., van Karnebeek, C., Vercauteren, S., Suri, M., Elmslie, F., Simon, M.E.H., van Gassen, K.L.I., Héron, D., Keren, B., Nava, C., Connolly, M.B., Demos, M., Farrer, M.J., 2017. De Novo Mutations in YWHAG Cause Early-Onset Epilepsy. Am. J. Hum. Genet. https://doi.org/10.1016/j.ajhg.2017.07.004

Jung, J., Kim, G.W., Lee, B., Joo, J.W.J., Jang, W., 2019. Integrative genomic and transcriptomic analysis of genetic markers in Dupuytren's disease. BMC Med. Genomics. https://doi.org/10.1186/s12920019-0518-3

Nam, K.N., Wolfe, C.M., Fitz, N.F., Letronne, F., Castranio, E.L., Mounier, A., Schug, J., Lefterov, I., Koldamova, R., 2018. Integrated approach reveals diet, APOE genotype and sex affect immune response in APP mice. Biochim. Biophys. Acta - Mol. Basis Dis. https://doi.org/10.1016/j.bbadis.2017.10.018

Schaefer, R.J., Briskine, R., Springer, N.M., Myers, C.L., 2014. Discovering functional modules across diverse maize transcriptomes using $\mathrm{COB}$, the co-expression browser. PLoS One. https://doi.org/10.1371/journal.pone.0099193

Shen, N., Heintz, C., Thiel, C., Okun, J.G., Hoffmann, G.F., Blau, N., 2016. Co-expression of phenylalanine hydroxylase variants and effects of interallelic complementation on in vitro enzyme activity and genotype-phenotype correlation. Mol. Genet. Metab. https://doi.org/10.1016/j.ymgme.2016.01.004

Veltmann, C., Barajas-Martinez, H., Wolpert, C., Borggrefe, M., Schimpf, R., Pfeiffer, R., Cáceres, G., Burashnikov, E., Antzelevitch, C., Hu, D., 2016. Further Insights in the Most Common SCN5A Mutation Causing Overlapping Phenotype of Long QT Syndrome, Brugada Syndrome, and Conduction Defect. J. Am. Heart Assoc. https://doi.org/10.1161/JAHA.116.003379

Yu, K. Da, Wang, X., Yang, C., Zeng, X.H., Shao, Z.M., 2015. Host genotype and tumor phenotype of chemokine decoy receptors integrally affect breast cancer relapse. Oncotarget. https://doi.org/10.18632/oncotarget.4470 


\section{Supplementary Material}

\section{Table S1. List of protein hormones related with human diseases}

\begin{tabular}{|c|c|c|}
\hline UniProt ID & Gene names & Protein names \\
\hline P01241 & GH1 & $\begin{array}{l}\text { Somatotropin (Growth hormone) (GH) (GH-N) (Growth } \\
\text { hormone 1) (Pituitary growth hormone) }\end{array}$ \\
\hline Q06187 & BTK AGMX1 ATK BPK & $\begin{array}{l}\text { Tyrosine-protein kinase BTK (EC 2.7.10.2) } \\
\text { (Agammaglobulinemia tyrosine kinase) (ATK) (B-cell } \\
\text { progenitor kinase) (BPK) (Bruton tyrosine kinase) }\end{array}$ \\
\hline P22888 & LHCGR LCGR LGR2 LHRHR & $\begin{array}{l}\text { Lutropin-choriogonadotropic hormone receptor (LH/CG-R) } \\
\text { (Luteinizing hormone receptor) (LHR) (LSH-R) }\end{array}$ \\
\hline P10828 & THRB ERBA2 NR1A2 THR1 & $\begin{array}{l}\text { Thyroid hormone receptor beta (Nuclear receptor subfamily } \\
1 \text { group A member 2) (c-erbA-2) (c-erbA-beta) }\end{array}$ \\
\hline P10912 & GHR & $\begin{array}{l}\text { Growth hormone receptor (GH receptor) (Somatotropin } \\
\text { receptor) [Cleaved into: Growth hormone-binding protein } \\
\text { (GH-binding protein) (GHBP) (Serum-binding protein)] }\end{array}$ \\
\hline Q96LT9 & $\begin{array}{l}\text { RNPC3 KIAA1839 RBM40 RNP } \\
\text { SNRNP65 }\end{array}$ & $\begin{array}{l}\text { RNA-binding region-containing protein } 3 \text { (RNA-binding motif } \\
\text { protein } 40 \text { ) (RNA-binding protein } 40 \text { ) (U11/U12 small nuclear } \\
\text { ribonucleoprotein } 65 \mathrm{kDa} \text { protein) (U11/U12 snRNP } 65 \mathrm{kDa} \\
\text { protein) (U11/U12-65K) }\end{array}$ \\
\hline Q9UBX0 & HESX1 HANF & $\begin{array}{l}\text { Homeobox expressed in ES cells } 1 \text { (Homeobox protein ANF) } \\
\text { (hAnf) }\end{array}$ \\
\hline Q02643 & GHRHR & $\begin{array}{l}\text { Growth hormone-releasing hormone receptor (GHRH } \\
\text { receptor) (Growth hormone-releasing factor receptor) (GRF } \\
\text { receptor) (GRFR) }\end{array}$ \\
\hline P51692 & STAT5B & Signal transducer and activator of transcription $5 \mathrm{~B}$ \\
\hline Q9NWF9 & $\begin{array}{l}\text { RNF216 TRIAD3 UBCE7IP1 } \\
\text { ZIN }\end{array}$ & $\begin{array}{l}\text { E3 ubiquitin-protein ligase RNF216 (EC 2.3.2.27) (RING finger } \\
\text { protein 216) (RING-type E3 ubiquitin transferase RNF216) } \\
\text { (Triad domain-containing protein 3) (Ubiquitin-conjugating } \\
\text { enzyme 7-interacting protein 1) (Zinc finger protein inhibiting } \\
\text { NF-kappa-B) }\end{array}$ \\
\hline P16473 & TSHR LGR3 & $\begin{array}{l}\text { Thyrotropin receptor (Thyroid-stimulating hormone } \\
\text { receptor) (TSH-R) }\end{array}$ \\
\hline Q969G2 & LHX4 & LIM/homeobox protein Lhx4 (LIM homeobox protein 4) \\
\hline P63092 & GNAS GNAS1 GSP & $\begin{array}{l}\text { Guanine nucleotide-binding protein G(s) subunit alpha } \\
\text { isoforms short (Adenylate cyclase-stimulating G alpha } \\
\text { protein) }\end{array}$ \\
\hline Q9UBR4 & LHX3 & LIM/homeobox protein Lhx3 (LIM homeobox protein 3) \\
\hline Q01718 & MC2R ACTHR & $\begin{array}{l}\text { Adrenocorticotropic hormone receptor (ACTH receptor) } \\
\text { (ACTH-R) (Adrenocorticotropin receptor) (Melanocortin } \\
\text { receptor 2) (MC2-R) }\end{array}$ \\
\hline P41225 & SOX3 & Transcription factor SOX-3 \\
\hline P07202 & TPO & Thyroid peroxidase (TPO) (EC 1.11.1.8) \\
\hline Q08499 & PDE4D DPDE3 & $\begin{array}{l}\text { cAMP-specific 3',5'-cyclic phosphodiesterase 4D (EC 3.1.4.53) } \\
\text { (DPDE3) (PDE43) }\end{array}$ \\
\hline P28069 & POU1F1 GHF1 PIT1 & $\begin{array}{l}\text { Pituitary-specific positive transcription factor } 1 \text { (PIT-1) } \\
\text { (Growth hormone factor 1) (GHF-1) }\end{array}$ \\
\hline
\end{tabular}




\begin{tabular}{|l|l|l|}
\hline P10644 & PRKAR1A PKR1 PRKAR1 TSE1 & $\begin{array}{l}\text { cAMP-dependent protein kinase type I-alpha regulatory } \\
\text { subunit (Tissue-specific extinguisher 1) (TSE1) }\end{array}$ \\
\hline Q06710 & PAX8 & Paired box protein Pax-8 \\
\hline 075360 & PROP1 & $\begin{array}{l}\text { Homeobox protein prophet of Pit-1 (PROP-1) (Pituitary- } \\
\text { specific homeodomain factor) }\end{array}$ \\
\hline P32243 & OTX2 & Homeobox protein OTX2 (Orthodenticle homolog 2) \\
\hline P84996 & GNAS GNAS1 & Protein ALEX (Alternative gene product encoded by XL-exon) \\
\hline 095467 & GNAS GNAS1 & $\begin{array}{l}\text { Neuroendocrine secretory protein 55 (NESP55) [Cleaved } \\
\text { into: LHAL tetrapeptide; GPIPIRRH peptide] }\end{array}$ \\
\hline 000170 & AIP XAP2 & $\begin{array}{l}\text { AH receptor-interacting protein (AIP) (Aryl-hydrocarbon } \\
\text { receptor-interacting protein) (HBV X-associated protein 2) } \\
\text { (XAP-2) (Immunophilin homolog ARA9) }\end{array}$ \\
\hline Q9NSE4 & IARS2 & $\begin{array}{l}\text { Isoleucine--tRNA ligase, mitochondrial (EC 6.1.1.5) (Isoleucyl- } \\
\text { tRNA synthetase) (lleRS) }\end{array}$ \\
\hline Q96T21 & SECISBP2 SBP2 & $\begin{array}{l}\text { Selenocysteine insertion sequence-binding protein 2 (SECIS- } \\
\text { binding protein 2) }\end{array}$ \\
\hline O60806 & TBX19 TPIT & $\begin{array}{l}\text { T-box transcription factor TBX19 (T-box protein 19) (T-box } \\
\text { factor, pituitary) }\end{array}$ \\
\hline Q96P66 & GPR101 & Probable G-protein coupled receptor 101 \\
\hline Q5JWF2 & GNAS GNAS1 & $\begin{array}{l}\text { Guanine nucleotide-binding protein G(s) subunit alpha } \\
\text { isoforms XLas (Adenylate cyclase-stimulating G alpha } \\
\text { protein) (Extra large alphas protein) (XLalphas) }\end{array}$ \\
\hline P01225 & FSHB & $\begin{array}{l}\text { Follitropin subunit beta (Follicle-stimulating hormone beta } \\
\text { subunit) (FSH-B) (FSH-beta) (Follitropin beta chain) }\end{array}$ \\
\hline Q92847 & GHSR & $\begin{array}{l}\text { Growth hormone secretagogue receptor type 1 (GHS-R) (GH- } \\
\text { releasing peptide receptor) (GHRP) (Ghrelin receptor) }\end{array}$ \\
\hline
\end{tabular}

Table S2. Protein pairs evaluated for co-expression

\begin{tabular}{|c|c|c|c|c|c|c|}
\hline $\begin{array}{c}\text { Pole1 } \\
\text { value }\end{array}$ & $\begin{array}{c}\text { Pole 2 } \\
\text { value }\end{array}$ & Protein 1 & Protein 2 & $\begin{array}{c}\text { Entrez } \\
\text { Gene ID } \\
\text { (Protein 1) }\end{array}$ & $\begin{array}{c}\text { Entrez } \\
\text { Gene ID } \\
\text { (Protein 2) }\end{array}$ & TEF \\
\hline-1.17026 & -0.40054 & P01241 & P01241 & 2688 & 2688 & 0.769719 \\
\hline-0.051 & -1.51979 & P01241 & Q96LT9 & 2688 & 55599 & 1.468793 \\
\hline-1.27733 & -0.29347 & P01241 & Q9UBX0 & 2688 & 8820 & 0.983862 \\
\hline-1.35639 & -0.21441 & P01241 & O75360 & 2688 & 5626 & 1.141974 \\
\hline-0.13031 & -1.44049 & P01241 & P84996 & 2688 & 2778 & 1.310186 \\
\hline-1.39153 & -0.17927 & P01241 & O95467 & 2688 & 2778 & 1.212254 \\
\hline-1.27752 & -0.29327 & P01241 & P01225 & 2688 & 2488 & 0.984248 \\
\hline-1.17026 & -0.40054 & Q06187 & Q06187 & 695 & 695 & 0.769719 \\
\hline-1.49988 & -0.07091 & Q06187 & Q02643 & 695 & 2692 & 1.428971 \\
\hline-1.48858 & -0.08221 & Q06187 & Q969G2 & 695 & 89884 & 1.40637 \\
\hline-1.54476 & -0.02603 & Q06187 & P41225 & 695 & 6658 & 1.518728 \\
\hline
\end{tabular}




\begin{tabular}{|c|c|c|c|c|c|c|}
\hline-1.49371 & -0.07709 & Q06187 & 000170 & 695 & 9049 & 1.416615 \\
\hline-1.17026 & -0.40054 & P22888 & P22888 & 3973 & 3973 & 0.769719 \\
\hline-1.56279 & -0.008 & P22888 & P63092 & 3973 & 2778 & 1.554793 \\
\hline-1.52627 & -0.04453 & P22888 & P41225 & 3973 & 6658 & 1.481737 \\
\hline-1.5675 & -0.00329 & P22888 & P32243 & 3973 & 5015 & 1.564211 \\
\hline-1.42112 & -0.14968 & P22888 & 000170 & 3973 & 9049 & 1.271445 \\
\hline-1.17026 & -0.40054 & P10828 & P10828 & 7068 & 7068 & 0.769719 \\
\hline-1.01609 & -0.5547 & P10828 & Q96LT9 & 7068 & 55599 & 0.461387 \\
\hline-0.15211 & -1.41869 & P10828 & P41225 & 7068 & 6658 & 1.266582 \\
\hline-0.01534 & -1.55545 & P10828 & Q06710 & 7068 & 7849 & 1.540108 \\
\hline-0.15456 & -1.41624 & P10828 & 060806 & 7068 & 9095 & 1.261683 \\
\hline-0.9163 & -0.6545 & P10828 & Q96P66 & 7068 & 83550 & 0.261799 \\
\hline-1.17026 & -0.40054 & P10912 & P10912 & 2690 & 2690 & 0.769719 \\
\hline-1.40011 & -0.17068 & P10912 & Q02643 & 2690 & 2692 & 1.229429 \\
\hline-1.52818 & -0.04262 & P10912 & Q9UBR4 & 2690 & 8022 & 1.485556 \\
\hline-0.051 & -1.51979 & Q96LT9 & P01241 & 55599 & 2688 & 1.468793 \\
\hline-1.01609 & -0.5547 & Q96LT9 & P10828 & 55599 & 7068 & 0.461387 \\
\hline-1.17026 & -0.40054 & Q96LT9 & Q96LT9 & 55599 & 55599 & 0.769719 \\
\hline-0.89293 & -0.67786 & Q96LT9 & Q02643 & 55599 & 2692 & 0.21507 \\
\hline-0.67979 & -0.89101 & Q96LT9 & Q969G2 & 55599 & 89884 & 0.211217 \\
\hline-0.74798 & -0.82282 & Q96LT9 & P63092 & 55599 & 2778 & 0.074841 \\
\hline-0.68684 & -0.88396 & Q96LT9 & Q9UBR4 & 55599 & 8022 & 0.197125 \\
\hline-0.38026 & -1.19053 & Q96LT9 & Q01718 & 55599 & 4158 & 0.81027 \\
\hline-1.04269 & -0.5281 & Q96LT9 & P41225 & 55599 & 6658 & 0.514587 \\
\hline-0.30355 & -1.26725 & Q96LT9 & P28069 & 55599 & 5449 & 0.963698 \\
\hline-0.52917 & -1.04163 & Q96LT9 & P10644 & 55599 & 5573 & 0.512458 \\
\hline-0.95831 & -0.61249 & Q96LT9 & Q06710 & 55599 & 7849 & 0.345822 \\
\hline-0.25782 & -1.31297 & Q96LT9 & P32243 & 55599 & 5015 & 1.055148 \\
\hline-0.32086 & -1.24993 & Q96LT9 & 000170 & 55599 & 9049 & 0.929069 \\
\hline-1.06735 & -0.50345 & Q96LT9 & 060806 & 55599 & 9095 & 0.563906 \\
\hline-1.34039 & -0.23041 & Q96LT9 & Q96P66 & 55599 & 83550 & 1.109984 \\
\hline-0.56463 & -1.00617 & Q96LT9 & Q92847 & 55599 & 2693 & 0.441542 \\
\hline-1.27733 & -0.29347 & Q9UBX0 & P01241 & 8820 & 2688 & 0.983862 \\
\hline-1.17026 & -0.40054 & Q9UBX0 & Q9UBX0 & 8820 & 8820 & 0.769719 \\
\hline-1.31708 & -0.25372 & Q9UBX0 & 075360 & 8820 & 5626 & 1.063354 \\
\hline-1.55272 & -0.01808 & Q9UBX0 & P32243 & 8820 & 5015 & 1.534634 \\
\hline-0.16936 & -1.40144 & Q9UBX0 & P84996 & 8820 & 2778 & 1.232084 \\
\hline-1.36777 & -0.20302 & Q9UBX0 & 095467 & 8820 & 2778 & 1.16475 \\
\hline-1.18667 & -0.38412 & Q9UBX0 & P01225 & 8820 & 2488 & 0.80255 \\
\hline-1.49988 & -0.07091 & Q02643 & Q06187 & 2692 & 695 & 1.428971 \\
\hline-1.40011 & -0.17068 & Q02643 & P10912 & 2692 & 2690 & 1.229429 \\
\hline
\end{tabular}




\begin{tabular}{|c|c|c|c|c|c|c|}
\hline-0.89293 & -0.67786 & Q02643 & Q96LT9 & 2692 & 55599 & 0.21507 \\
\hline-1.17026 & -0.40054 & Q02643 & Q02643 & 2692 & 2692 & 0.769719 \\
\hline-1.29816 & -0.27264 & Q02643 & P84996 & 2692 & 2778 & 1.025521 \\
\hline-0.68185 & -0.88895 & Q02643 & Q96P66 & 2692 & 83550 & 0.207094 \\
\hline-1.17026 & -0.40054 & P51692 & P51692 & 6777 & 6777 & 0.769719 \\
\hline-1.17026 & -0.40054 & Q9NWF9 & Q9NWF9 & 54476 & 54476 & 0.769719 \\
\hline-1.17026 & -0.40054 & P16473 & P16473 & 7253 & 7253 & 0.769719 \\
\hline-1.48858 & -0.08221 & Q969G2 & Q06187 & 89884 & 695 & 1.40637 \\
\hline-0.67979 & -0.89101 & Q969G2 & Q96LT9 & 89884 & 55599 & 0.211217 \\
\hline-1.17026 & -0.40054 & Q969G2 & Q969G2 & 89884 & 89884 & 0.769719 \\
\hline-1.52478 & -0.04602 & Q969G2 & P84996 & 89884 & 2778 & 1.478757 \\
\hline-0.0664 & -1.5044 & Q969G2 & Q96P66 & 89884 & 83550 & 1.438004 \\
\hline-1.56279 & -0.008 & P63092 & P22888 & 2778 & 3973 & 1.554793 \\
\hline-0.74798 & -0.82282 & P63092 & Q96LT9 & 2778 & 55599 & 0.074841 \\
\hline-1.17026 & -0.40054 & P63092 & P63092 & 2778 & 2778 & 0.769719 \\
\hline-1.22312 & -0.34767 & P63092 & P84996 & 2778 & 2778 & 0.875451 \\
\hline-0.53474 & -1.03605 & P63092 & Q96P66 & 2778 & 83550 & 0.501312 \\
\hline-1.52818 & -0.04262 & Q9UBR4 & P10912 & 8022 & 2690 & 1.485556 \\
\hline-0.68684 & -0.88396 & Q9UBR4 & Q96LT9 & 8022 & 55599 & 0.197125 \\
\hline-1.17026 & -0.40054 & Q9UBR4 & Q9UBR4 & 8022 & 8022 & 0.769719 \\
\hline-0.29883 & -1.27196 & Q9UBR4 & Q96P66 & 8022 & 83550 & 0.973131 \\
\hline-0.38026 & -1.19053 & Q01718 & Q96LT9 & 4158 & 55599 & 0.81027 \\
\hline-1.17026 & -0.40054 & Q01718 & Q01718 & 4158 & 4158 & 0.769719 \\
\hline-1.08791 & -0.48289 & Q01718 & P84996 & 4158 & 2778 & 0.605023 \\
\hline-1.54476 & -0.02603 & P41225 & Q06187 & 6658 & 695 & 1.518728 \\
\hline-1.52627 & -0.04453 & P41225 & P22888 & 6658 & 3973 & 1.481737 \\
\hline-0.15211 & -1.41869 & P41225 & P10828 & 6658 & 7068 & 1.266582 \\
\hline-1.04269 & -0.5281 & P41225 & Q96LT9 & 6658 & 55599 & 0.514587 \\
\hline-1.17026 & -0.40054 & P41225 & P41225 & 6658 & 6658 & 0.769719 \\
\hline-0.73004 & -0.84076 & P41225 & Q96P66 & 6658 & 83550 & 0.110717 \\
\hline-1.17026 & -0.40054 & P07202 & P07202 & 7173 & 7173 & 0.769719 \\
\hline-1.17026 & -0.40054 & Q08499 & Q08499 & 5144 & 5144 & 0.769719 \\
\hline-0.30355 & -1.26725 & P28069 & Q96LT9 & 5449 & 55599 & 0.963698 \\
\hline-1.17026 & -0.40054 & P28069 & P28069 & 5449 & 5449 & 0.769719 \\
\hline-0.03466 & -1.53613 & P28069 & Q96P66 & 5449 & 83550 & 1.501472 \\
\hline-0.52917 & -1.04163 & P10644 & Q96LT9 & 5573 & 55599 & 0.512458 \\
\hline-1.17026 & -0.40054 & P10644 & P10644 & 5573 & 5573 & 0.769719 \\
\hline-1.24418 & -0.32662 & P10644 & P84996 & 5573 & 2778 & 0.917556 \\
\hline-0.3803 & -1.19049 & P10644 & Q96P66 & 5573 & 83550 & 0.810187 \\
\hline-0.01534 & -1.55545 & Q06710 & P10828 & 7849 & 7068 & 1.540108 \\
\hline-0.95831 & -0.61249 & Q06710 & Q96LT9 & 7849 & 55599 & 0.345822 \\
\hline
\end{tabular}




\begin{tabular}{|c|c|c|c|c|c|c|}
\hline-1.17026 & -0.40054 & Q06710 & Q06710 & 7849 & 7849 & 0.769719 \\
\hline-0.95108 & -0.61972 & Q06710 & Q96P66 & 7849 & 83550 & 0.331359 \\
\hline-1.35639 & -0.21441 & 075360 & P01241 & 5626 & 2688 & 1.141974 \\
\hline-1.31708 & -0.25372 & 075360 & Q9UBX0 & 5626 & 8820 & 1.063354 \\
\hline-1.17026 & -0.40054 & 075360 & 075360 & 5626 & 5626 & 0.769719 \\
\hline-0.08104 & -1.48975 & 075360 & P84996 & 5626 & 2778 & 1.408711 \\
\hline-1.42831 & -0.14249 & 075360 & 095467 & 5626 & 2778 & 1.285814 \\
\hline-1.31761 & -0.25319 & 075360 & P01225 & 5626 & 2488 & 1.064418 \\
\hline-1.5675 & -0.00329 & P32243 & P22888 & 5015 & 3973 & 1.564211 \\
\hline-0.25782 & -1.31297 & P32243 & Q96LT9 & 5015 & 55599 & 1.055148 \\
\hline-1.55272 & -0.01808 & P32243 & Q9UBX0 & 5015 & 8820 & 1.534634 \\
\hline-1.17026 & -0.40054 & P32243 & P32243 & 5015 & 5015 & 0.769719 \\
\hline-1.3002 & -0.2706 & P32243 & P84996 & 5015 & 2778 & 1.029595 \\
\hline-0.01016 & -1.56063 & P32243 & Q96P66 & 5015 & 83550 & 1.550472 \\
\hline-1.5554 & -0.0154 & P32243 & P01225 & 5015 & 2488 & 1.539998 \\
\hline-0.13031 & -1.44049 & P84996 & P01241 & 2778 & 2688 & 1.310186 \\
\hline-0.16936 & -1.40144 & P84996 & Q9UBX0 & 2778 & 8820 & 1.232084 \\
\hline-1.29816 & -0.27264 & P84996 & Q02643 & 2778 & 2692 & 1.025521 \\
\hline-1.52478 & -0.04602 & P84996 & Q969G2 & 2778 & 89884 & 1.478757 \\
\hline-1.22312 & -0.34767 & P84996 & P63092 & 2778 & 2778 & 0.875451 \\
\hline-1.08791 & -0.48289 & P84996 & Q01718 & 2778 & 4158 & 0.605023 \\
\hline-1.24418 & -0.32662 & P84996 & P10644 & 2778 & 5573 & 0.917556 \\
\hline-0.08104 & -1.48975 & P84996 & 075360 & 2778 & 5626 & 1.408711 \\
\hline-1.3002 & -0.2706 & P84996 & P32243 & 2778 & 5015 & 1.029595 \\
\hline-1.17026 & -0.40054 & P84996 & P84996 & 2778 & 2778 & 0.769719 \\
\hline-0.14384 & -1.42696 & P84996 & 095467 & 2778 & 2778 & 1.28312 \\
\hline-1.2524 & -0.3184 & P84996 & 000170 & 2778 & 9049 & 0.934004 \\
\hline-1.53964 & -0.03116 & P84996 & 060806 & 2778 & 9095 & 1.50848 \\
\hline-0.18606 & -1.38474 & P84996 & P01225 & 2778 & 2488 & 1.198684 \\
\hline-1.39153 & -0.17927 & 095467 & P01241 & 2778 & 2688 & 1.212254 \\
\hline-1.36777 & -0.20302 & 095467 & Q9UBX0 & 2778 & 8820 & 1.16475 \\
\hline-1.42831 & -0.14249 & 095467 & 075360 & 2778 & 5626 & 1.285814 \\
\hline-0.14384 & -1.42696 & 095467 & P84996 & 2778 & 2778 & 1.28312 \\
\hline-1.17026 & -0.40054 & 095467 & 095467 & 2778 & 2778 & 0.769719 \\
\hline-1.36645 & -0.20434 & 095467 & P01225 & 2778 & 2488 & 1.162111 \\
\hline-1.49371 & -0.07709 & 000170 & Q06187 & 9049 & 695 & 1.416615 \\
\hline-1.42112 & -0.14968 & 000170 & P22888 & 9049 & 3973 & 1.271445 \\
\hline-0.32086 & -1.24993 & 000170 & Q96LT9 & 9049 & 55599 & 0.929069 \\
\hline-1.2524 & -0.3184 & 000170 & P84996 & 9049 & 2778 & 0.934004 \\
\hline-1.17026 & -0.40054 & 000170 & 000170 & 9049 & 9049 & 0.769719 \\
\hline-0.01262 & -1.55818 & 000170 & Q96P66 & 9049 & 83550 & 1.545563 \\
\hline
\end{tabular}




\begin{tabular}{|c|c|c|c|c|c|c|}
\hline-1.17026 & -0.40054 & Q9NSE4 & Q9NSE4 & 55699 & 55699 & 0.769719 \\
\hline-1.17026 & -0.40054 & Q96T21 & Q96T21 & 79048 & 79048 & 0.769719 \\
\hline-0.15456 & -1.41624 & 060806 & P10828 & 9095 & 7068 & 1.261683 \\
\hline-1.06735 & -0.50345 & 060806 & Q96LT9 & 9095 & 55599 & 0.563906 \\
\hline-1.53964 & -0.03116 & 060806 & P84996 & 9095 & 2778 & 1.50848 \\
\hline-1.17026 & -0.40054 & 060806 & 060806 & 9095 & 9095 & 0.769719 \\
\hline-0.99764 & -0.57315 & 060806 & Q96P66 & 9095 & 83550 & 0.42449 \\
\hline-0.9163 & -0.6545 & Q96P66 & P10828 & 83550 & 7068 & 0.261799 \\
\hline-1.34039 & -0.23041 & Q96P66 & Q96LT9 & 83550 & 55599 & 1.109984 \\
\hline-0.68185 & -0.88895 & Q96P66 & Q02643 & 83550 & 2692 & 0.207094 \\
\hline-0.0664 & -1.5044 & Q96P66 & Q969G2 & 83550 & 89884 & 1.438004 \\
\hline-0.53474 & -1.03605 & Q96P66 & P63092 & 83550 & 2778 & 0.501312 \\
\hline-0.29883 & -1.27196 & Q96P66 & Q9UBR4 & 83550 & 8022 & 0.973131 \\
\hline-0.73004 & -0.84076 & Q96P66 & P41225 & 83550 & 6658 & 0.110717 \\
\hline-0.03466 & -1.53613 & Q96P66 & P28069 & 83550 & 5449 & 1.501472 \\
\hline-0.3803 & -1.19049 & Q96P66 & P10644 & 83550 & 5573 & 0.810187 \\
\hline-0.95108 & -0.61972 & Q96P66 & Q06710 & 83550 & 7849 & 0.331359 \\
\hline-0.01016 & -1.56063 & Q96P66 & P32243 & 83550 & 5015 & 1.550472 \\
\hline-0.01262 & -1.55818 & Q96P66 & 000170 & 83550 & 9049 & 1.545563 \\
\hline-0.99764 & -0.57315 & Q96P66 & 060806 & 83550 & 9095 & 0.42449 \\
\hline-1.17026 & -0.40054 & Q96P66 & Q96P66 & 83550 & 83550 & 0.769719 \\
\hline-0.14152 & -1.42928 & Q96P66 & Q92847 & 83550 & 2693 & 1.287758 \\
\hline-1.17026 & -0.40054 & Q5JWF2 & Q5JWF2 & 2778 & 2778 & 0.769719 \\
\hline-1.27752 & -0.29327 & P01225 & P01241 & 2488 & 2688 & 0.984248 \\
\hline-1.18667 & -0.38412 & P01225 & Q9UBX0 & 2488 & 8820 & 0.80255 \\
\hline-1.31761 & -0.25319 & P01225 & 075360 & 2488 & 5626 & 1.064418 \\
\hline-1.5554 & -0.0154 & P01225 & P32243 & 2488 & 5015 & 1.539998 \\
\hline-0.18606 & -1.38474 & P01225 & P84996 & 2488 & 2778 & 1.198684 \\
\hline-1.36645 & -0.20434 & P01225 & 095467 & 2488 & 2778 & 1.162111 \\
\hline-1.17026 & -0.40054 & P01225 & P01225 & 2488 & 2488 & 0.769719 \\
\hline-0.56463 & -1.00617 & Q92847 & Q96LT9 & 2693 & 55599 & 0.441542 \\
\hline-0.14152 & -1.42928 & Q92847 & Q96P66 & 2693 & 83550 & 1.287758 \\
\hline-1.17026 & -0.40054 & Q92847 & Q92847 & 2693 & 2693 & 0.769719 \\
\hline 1.373299 & 0.197497 & P01241 & Q06187 & 2688 & 695 & 1.175802 \\
\hline 1.379417 & 0.19138 & P01241 & P22888 & 2688 & 3973 & 1.188037 \\
\hline 0.901387 & 0.669409 & P01241 & P10828 & 2688 & 7068 & 0.231978 \\
\hline 1.450649 & 0.120147 & P01241 & P10912 & 2688 & 2690 & 1.330501 \\
\hline 1.086596 & 0.484201 & P01241 & Q02643 & 2688 & 2692 & 0.602395 \\
\hline 1.35591 & 0.214886 & P01241 & P51692 & 2688 & 6777 & 1.141024 \\
\hline 1.232911 & 0.337885 & P01241 & Q9NWF9 & 2688 & 54476 & 0.895026 \\
\hline 1.300964 & 0.269832 & P01241 & P16473 & 2688 & 7253 & 1.031132 \\
\hline
\end{tabular}




\begin{tabular}{|c|c|c|c|c|c|c|}
\hline 1.060278 & 0.510519 & P01241 & Q969G2 & 2688 & 89884 & 0.549759 \\
\hline 1.107344 & 0.463453 & P01241 & P63092 & 2688 & 2778 & 0.643891 \\
\hline 1.249639 & 0.321157 & $\mathrm{P} 01241$ & Q9UBR4 & 2688 & 8022 & 0.928482 \\
\hline 1.478867 & 0.091929 & P01241 & Q01718 & 2688 & 4158 & 1.386938 \\
\hline 1.090885 & 0.479912 & P01241 & P41225 & 2688 & 6658 & 0.610973 \\
\hline 1.338789 & 0.232007 & $\mathrm{P} 01241$ & P07202 & 2688 & 7173 & 1.106782 \\
\hline 1.273813 & 0.296983 & P01241 & Q08499 & 2688 & 5144 & 0.97683 \\
\hline 1.492543 & 0.078254 & P01241 & P28069 & 2688 & 5449 & 1.414289 \\
\hline 1.210249 & 0.360547 & P01241 & P10644 & 2688 & 5573 & 0.849702 \\
\hline 1.289006 & 0.28179 & $\mathrm{P} 01241$ & Q06710 & 2688 & 7849 & 1.007216 \\
\hline 1.547491 & 0.023305 & P01241 & P32243 & 2688 & 5015 & 1.524186 \\
\hline 1.277555 & 0.293242 & $\mathrm{P} 01241$ & 000170 & 2688 & 9049 & 0.984313 \\
\hline 1.264313 & 0.306483 & P01241 & Q9NSE4 & 2688 & 55699 & 0.95783 \\
\hline 1.196012 & 0.374785 & $\mathrm{P} 01241$ & Q96T21 & 2688 & 79048 & 0.821227 \\
\hline 1.040637 & 0.530159 & P01241 & 060806 & 2688 & 9095 & 0.510478 \\
\hline 0.262568 & 1.308228 & P01241 & Q96P66 & 2688 & 83550 & 1.045661 \\
\hline 1.267266 & 0.303531 & $\mathrm{P} 01241$ & Q5JWF2 & 2688 & 2778 & 0.963735 \\
\hline 1.184614 & 0.386182 & P01241 & Q92847 & 2688 & 2693 & 0.798433 \\
\hline 1.373299 & 0.197497 & Q06187 & P01241 & 695 & 2688 & 1.175802 \\
\hline 1.316992 & 0.253804 & Q06187 & P22888 & 695 & 3973 & 1.063188 \\
\hline 1.50043 & 0.070366 & Q06187 & P10828 & 695 & 7068 & 1.430064 \\
\hline 1.34857 & 0.222227 & Q06187 & P10912 & 695 & 2690 & 1.126343 \\
\hline 1.431283 & 0.139513 & Q06187 & Q96LT9 & 695 & 55599 & 1.29177 \\
\hline 1.401261 & 0.169535 & Q06187 & Q9UBX0 & 695 & 8820 & 1.231726 \\
\hline 1.296046 & 0.27475 & Q06187 & P51692 & 695 & 6777 & 1.021296 \\
\hline 1.361415 & 0.209382 & Q06187 & Q9NWF9 & 695 & 54476 & 1.152033 \\
\hline 1.404128 & 0.166669 & Q06187 & P16473 & 695 & 7253 & 1.237459 \\
\hline 1.418896 & 0.1519 & Q06187 & P63092 & 695 & 2778 & 1.266995 \\
\hline 1.542638 & 0.028159 & Q06187 & Q9UBR4 & 695 & 8022 & 1.514479 \\
\hline 1.470784 & 0.100012 & Q06187 & Q01718 & 695 & 4158 & 1.370772 \\
\hline 1.276269 & 0.294527 & Q06187 & P07202 & 695 & 7173 & 0.981742 \\
\hline 1.242438 & 0.328359 & Q06187 & Q08499 & 695 & 5144 & 0.914079 \\
\hline 1.42306 & 0.147736 & Q06187 & P28069 & 695 & 5449 & 1.275324 \\
\hline 1.509098 & 0.061698 & Q06187 & P10644 & 695 & 5573 & 1.4474 \\
\hline 1.463946 & 0.10685 & Q06187 & Q06710 & 695 & 7849 & 1.357096 \\
\hline 1.450077 & 0.120719 & Q06187 & 075360 & 695 & 5626 & 1.329358 \\
\hline 1.530523 & 0.040273 & Q06187 & P32243 & 695 & 5015 & 1.490251 \\
\hline 1.476387 & 0.094409 & Q06187 & P84996 & 695 & 2778 & 1.381978 \\
\hline 1.361859 & 0.208938 & Q06187 & 095467 & 695 & 2778 & 1.152921 \\
\hline 1.287082 & 0.283715 & Q06187 & Q9NSE4 & 695 & 55699 & 1.003367 \\
\hline 1.325282 & 0.245515 & Q06187 & Q96T21 & 695 & 79048 & 1.079767 \\
\hline
\end{tabular}




\begin{tabular}{|c|c|c|c|c|c|c|}
\hline 1.425578 & 0.145219 & Q06187 & 060806 & 695 & 9095 & 1.280359 \\
\hline 1.524339 & 0.046458 & Q06187 & Q96P66 & 695 & 83550 & 1.477881 \\
\hline 1.272306 & 0.29849 & Q06187 & Q5JWF2 & 695 & 2778 & 0.973815 \\
\hline 1.41773 & 0.153066 & Q06187 & P01225 & 695 & 2488 & 1.264664 \\
\hline 1.405704 & 0.165092 & Q06187 & Q92847 & 695 & 2693 & 1.240612 \\
\hline 1.379417 & 0.19138 & P22888 & P01241 & 3973 & 2688 & 1.188037 \\
\hline 1.316992 & 0.253804 & P22888 & Q06187 & 3973 & 695 & 1.063188 \\
\hline 1.478466 & 0.09233 & P22888 & P10828 & 3973 & 7068 & 1.386136 \\
\hline 1.405014 & 0.165782 & P22888 & P10912 & 3973 & 2690 & 1.239233 \\
\hline 1.347204 & 0.223592 & P22888 & Q96LT9 & 3973 & 55599 & 1.123612 \\
\hline 1.363203 & 0.207593 & P22888 & Q9UBX0 & 3973 & 8820 & 1.15561 \\
\hline 1.422872 & 0.147925 & P22888 & Q02643 & 3973 & 2692 & 1.274947 \\
\hline 1.369698 & 0.201098 & P22888 & P51692 & 3973 & 6777 & 1.1686 \\
\hline 1.4176 & 0.153196 & P22888 & Q9NWF9 & 3973 & 54476 & 1.264404 \\
\hline 1.420601 & 0.150195 & P22888 & P16473 & 3973 & 7253 & 1.270406 \\
\hline 1.447404 & 0.123393 & P22888 & Q969G2 & 3973 & 89884 & 1.324011 \\
\hline 1.537469 & 0.033327 & P22888 & Q9UBR4 & 3973 & 8022 & 1.504141 \\
\hline 1.440933 & 0.129863 & P22888 & Q01718 & 3973 & 4158 & 1.31107 \\
\hline 1.307605 & 0.263191 & P22888 & P07202 & 3973 & 7173 & 1.044414 \\
\hline 1.320309 & 0.250487 & P22888 & Q08499 & 3973 & 5144 & 1.069822 \\
\hline 1.453773 & 0.117024 & P22888 & P28069 & 3973 & 5449 & 1.336749 \\
\hline 1.46422 & 0.106576 & P22888 & P10644 & 3973 & 5573 & 1.357643 \\
\hline 1.46447 & 0.106326 & P22888 & Q06710 & 3973 & 7849 & 1.358145 \\
\hline 1.48284 & 0.087957 & P22888 & 075360 & 3973 & 5626 & 1.394883 \\
\hline 1.245642 & 0.325154 & P22888 & P84996 & 3973 & 2778 & 0.920488 \\
\hline 1.336192 & 0.234604 & P22888 & 095467 & 3973 & 2778 & 1.101589 \\
\hline 1.295711 & 0.275086 & P22888 & Q9NSE4 & 3973 & 55699 & 1.020625 \\
\hline 1.422595 & 0.148202 & P22888 & Q96T21 & 3973 & 79048 & 1.274393 \\
\hline 1.55498 & 0.015816 & P22888 & 060806 & 3973 & 9095 & 1.539164 \\
\hline 1.427758 & 0.143039 & P22888 & Q96P66 & 3973 & 83550 & 1.284719 \\
\hline 1.340824 & 0.229973 & P22888 & Q5JWF2 & 3973 & 2778 & 1.110851 \\
\hline 1.34683 & 0.223966 & P22888 & P01225 & 3973 & 2488 & 1.122864 \\
\hline 1.40101 & 0.169786 & P22888 & Q92847 & 3973 & 2693 & 1.231224 \\
\hline 0.901387 & 0.669409 & P10828 & P01241 & 7068 & 2688 & 0.231978 \\
\hline 1.50043 & 0.070366 & P10828 & Q06187 & 7068 & 695 & 1.430064 \\
\hline 1.478466 & 0.09233 & P10828 & P22888 & 7068 & 3973 & 1.386136 \\
\hline 1.469274 & 0.101522 & P10828 & P10912 & 7068 & 2690 & 1.367752 \\
\hline 0.997342 & 0.573454 & P10828 & Q9UBX0 & 7068 & 8820 & 0.423888 \\
\hline 0.02122 & 1.549576 & P10828 & Q02643 & 7068 & 2692 & 1.528356 \\
\hline 1.498072 & 0.072725 & P10828 & P51692 & 7068 & 6777 & 1.425347 \\
\hline 1.32247 & 0.248327 & P10828 & Q9NWF9 & 7068 & 54476 & 1.074143 \\
\hline
\end{tabular}




\begin{tabular}{|c|c|c|c|c|c|c|}
\hline 1.459509 & 0.111287 & P10828 & P16473 & 7068 & 7253 & 1.348222 \\
\hline 0.288807 & 1.281989 & P10828 & Q969G2 & 7068 & 89884 & 0.993182 \\
\hline 0.222237 & 1.348559 & P10828 & P63092 & 7068 & 2778 & 1.126322 \\
\hline 0.449477 & 1.12132 & P10828 & Q9UBR4 & 7068 & 8022 & 0.671843 \\
\hline 0.774031 & 0.796766 & P10828 & Q01718 & 7068 & 4158 & 0.022735 \\
\hline 1.262976 & 0.30782 & P10828 & P07202 & 7068 & 7173 & 0.955156 \\
\hline 1.398481 & 0.172315 & P10828 & Q08499 & 7068 & 5144 & 1.226166 \\
\hline 0.795252 & 0.775545 & P10828 & P28069 & 7068 & 5449 & 0.019707 \\
\hline 0.398144 & 1.172652 & P10828 & P10644 & 7068 & 5573 & 0.774507 \\
\hline 1.087061 & 0.483735 & P10828 & 075360 & 7068 & 5626 & 0.603326 \\
\hline 0.706874 & 0.863922 & P10828 & P32243 & 7068 & 5015 & 0.157048 \\
\hline 1.513465 & 0.057331 & P10828 & P84996 & 7068 & 2778 & 1.456134 \\
\hline 0.858138 & 0.712658 & P10828 & 095467 & 7068 & 2778 & 0.14548 \\
\hline 0.493149 & 1.077647 & P10828 & 000170 & 7068 & 9049 & 0.584497 \\
\hline 1.239478 & 0.331318 & P10828 & Q9NSE4 & 7068 & 55699 & 0.90816 \\
\hline 1.339856 & 0.23094 & P10828 & Q96T21 & 7068 & 79048 & 1.108916 \\
\hline 1.32569 & 0.245106 & P10828 & Q5JWF2 & 7068 & 2778 & 1.080584 \\
\hline 0.980467 & 0.590329 & P10828 & P01225 & 7068 & 2488 & 0.390138 \\
\hline 0.325755 & 1.245042 & P10828 & Q92847 & 7068 & 2693 & 0.919287 \\
\hline 1.450649 & 0.120147 & P10912 & P01241 & 2690 & 2688 & 1.330501 \\
\hline 1.34857 & 0.222227 & P10912 & Q06187 & 2690 & 695 & 1.126343 \\
\hline 1.405014 & 0.165782 & P10912 & P22888 & 2690 & 3973 & 1.239233 \\
\hline 1.469274 & 0.101522 & P10912 & P10828 & 2690 & 7068 & 1.367752 \\
\hline 1.334921 & 0.235875 & P10912 & Q96LT9 & 2690 & 55599 & 1.099046 \\
\hline 1.416755 & 0.154042 & P10912 & Q9UBX0 & 2690 & 8820 & 1.262713 \\
\hline 1.383255 & 0.187542 & P10912 & P51692 & 2690 & 6777 & 1.195713 \\
\hline 1.368187 & 0.202609 & P10912 & Q9NWF9 & 2690 & 54476 & 1.165579 \\
\hline 1.25292 & 0.317876 & P10912 & P16473 & 2690 & 7253 & 0.935045 \\
\hline 1.437197 & 0.133599 & P10912 & Q969G2 & 2690 & 89884 & 1.303599 \\
\hline 1.506426 & 0.06437 & P10912 & P63092 & 2690 & 2778 & 1.442056 \\
\hline 1.370689 & 0.200108 & P10912 & Q01718 & 2690 & 4158 & 1.170581 \\
\hline 1.239331 & 0.331465 & P10912 & P41225 & 2690 & 6658 & 0.907866 \\
\hline 1.361993 & 0.208803 & P10912 & P07202 & 2690 & 7173 & 1.15319 \\
\hline 1.46161 & 0.109187 & P10912 & Q08499 & 2690 & 5144 & 1.352423 \\
\hline 1.539218 & 0.031579 & P10912 & P28069 & 2690 & 5449 & 1.507639 \\
\hline 1.434003 & 0.136793 & P10912 & P10644 & 2690 & 5573 & 1.29721 \\
\hline 1.450286 & 0.12051 & P10912 & Q06710 & 2690 & 7849 & 1.329776 \\
\hline 1.369798 & 0.200999 & P10912 & 075360 & 2690 & 5626 & 1.168799 \\
\hline 1.552789 & 0.018007 & P10912 & P32243 & 2690 & 5015 & 1.534782 \\
\hline 1.391804 & 0.178993 & P10912 & P84996 & 2690 & 2778 & 1.212811 \\
\hline 1.482129 & 0.088667 & P10912 & 095467 & 2690 & 2778 & 1.393462 \\
\hline
\end{tabular}




\begin{tabular}{|c|c|c|c|c|c|c|}
\hline 1.531827 & 0.038969 & P10912 & 000170 & 2690 & 9049 & 1.492859 \\
\hline 1.315406 & 0.25539 & P10912 & Q9NSE4 & 2690 & 55699 & 1.060016 \\
\hline 1.318239 & 0.252557 & P10912 & Q96T21 & 2690 & 79048 & 1.065682 \\
\hline 1.414154 & 0.156643 & P10912 & 060806 & 2690 & 9095 & 1.257511 \\
\hline 1.449864 & 0.120933 & P10912 & Q96P66 & 2690 & 83550 & 1.328931 \\
\hline 1.211346 & 0.359451 & P10912 & Q5JWF2 & 2690 & 2778 & 0.851895 \\
\hline 1.399484 & 0.171312 & P10912 & P01225 & 2690 & 2488 & 1.228172 \\
\hline 1.429716 & 0.141081 & P10912 & Q92847 & 2690 & 2693 & 1.288635 \\
\hline 1.431283 & 0.139513 & Q96LT9 & Q06187 & 55599 & 695 & 1.29177 \\
\hline 1.347204 & 0.223592 & Q96LT9 & P22888 & 55599 & 3973 & 1.123612 \\
\hline 1.334921 & 0.235875 & Q96LT9 & P10912 & 55599 & 2690 & 1.099046 \\
\hline 0.210896 & 1.3599 & Q96LT9 & Q9UBX0 & 55599 & 8820 & 1.149004 \\
\hline 1.465641 & 0.105155 & Q96LT9 & P51692 & 55599 & 6777 & 1.360486 \\
\hline 1.346372 & 0.224424 & Q96LT9 & Q9NWF9 & 55599 & 54476 & 1.121948 \\
\hline 1.375672 & 0.195124 & Q96LT9 & P16473 & 55599 & 7253 & 1.180548 \\
\hline 1.362634 & 0.208162 & Q96LT9 & P07202 & 55599 & 7173 & 1.154473 \\
\hline 1.344559 & 0.226238 & Q96LT9 & Q08499 & 55599 & 5144 & 1.118321 \\
\hline 0.010763 & 1.560033 & Q96LT9 & 075360 & 55599 & 5626 & 1.54927 \\
\hline 1.386692 & 0.184105 & Q96LT9 & P84996 & 55599 & 2778 & 1.202587 \\
\hline 0.590527 & 0.980269 & Q96LT9 & 095467 & 55599 & 2778 & 0.389743 \\
\hline 1.4731 & 0.097696 & Q96LT9 & Q9NSE4 & 55599 & 55699 & 1.375404 \\
\hline 1.332388 & 0.238409 & Q96LT9 & Q96T21 & 55599 & 79048 & 1.093979 \\
\hline 1.45071 & 0.120086 & Q96LT9 & Q5JWF2 & 55599 & 2778 & 1.330624 \\
\hline 0.270143 & 1.300653 & Q96LT9 & P01225 & 55599 & 2488 & 1.03051 \\
\hline 1.401261 & 0.169535 & Q9UBX0 & Q06187 & 8820 & 695 & 1.231726 \\
\hline 1.363203 & 0.207593 & Q9UBX0 & P22888 & 8820 & 3973 & 1.15561 \\
\hline 0.997342 & 0.573454 & Q9UBX0 & P10828 & 8820 & 7068 & 0.423888 \\
\hline 1.416755 & 0.154042 & Q9UBX0 & P10912 & 8820 & 2690 & 1.262713 \\
\hline 0.210896 & 1.3599 & Q9UBX0 & Q96LT9 & 8820 & 55599 & 1.149004 \\
\hline 1.114238 & 0.456558 & Q9UBX0 & Q02643 & 8820 & 2692 & 0.657681 \\
\hline 1.396734 & 0.174062 & Q9UBX0 & P51692 & 8820 & 6777 & 1.222672 \\
\hline 1.299569 & 0.271227 & Q9UBX0 & Q9NWF9 & 8820 & 54476 & 1.028342 \\
\hline 1.444509 & 0.126287 & Q9UBX0 & P16473 & 8820 & 7253 & 1.318222 \\
\hline 1.152866 & 0.41793 & Q9UBX0 & Q969G2 & 8820 & 89884 & 0.734935 \\
\hline 1.083316 & 0.48748 & Q9UBX0 & P63092 & 8820 & 2778 & 0.595836 \\
\hline 1.320472 & 0.250324 & Q9UBX0 & Q9UBR4 & 8820 & 8022 & 1.070149 \\
\hline 1.519538 & 0.051258 & Q9UBX0 & Q01718 & 8820 & 4158 & 1.46828 \\
\hline 1.167718 & 0.403078 & Q9UBX0 & P41225 & 8820 & 6658 & 0.76464 \\
\hline 1.323535 & 0.247261 & Q9UBX0 & P07202 & 8820 & 7173 & 1.076274 \\
\hline 1.311002 & 0.259794 & Q9UBX0 & Q08499 & 8820 & 5144 & 1.051207 \\
\hline 1.521566 & 0.04923 & Q9UBX0 & P28069 & 8820 & 5449 & 1.472336 \\
\hline
\end{tabular}




\begin{tabular}{|c|c|c|c|c|c|c|}
\hline 1.242879 & 0.327917 & Q9UBX0 & P10644 & 8820 & 5573 & 0.914962 \\
\hline 1.362044 & 0.208753 & Q9UBX0 & Q06710 & 8820 & 7849 & 1.153291 \\
\hline 1.301202 & 0.269594 & Q9UBX0 & 000170 & 8820 & 9049 & 1.031608 \\
\hline 1.226141 & 0.344655 & Q9UBX0 & Q9NSE4 & 8820 & 55699 & 0.881485 \\
\hline 1.208142 & 0.362655 & Q9UBX0 & Q96T21 & 8820 & 79048 & 0.845487 \\
\hline 1.061803 & 0.508993 & Q9UBX0 & 060806 & 8820 & 9095 & 0.55281 \\
\hline 0.20086 & 1.369936 & Q9UBX0 & Q96P66 & 8820 & 83550 & 1.169076 \\
\hline 1.236102 & 0.334694 & Q9UBX0 & Q5JWF2 & 8820 & 2778 & 0.901408 \\
\hline 1.241191 & 0.329606 & Q9UBX0 & Q92847 & 8820 & 2693 & 0.911585 \\
\hline 1.086596 & 0.484201 & Q02643 & P01241 & 2692 & 2688 & 0.602395 \\
\hline 1.422872 & 0.147925 & Q02643 & P22888 & 2692 & 3973 & 1.274947 \\
\hline 0.02122 & 1.549576 & Q02643 & P10828 & 2692 & 7068 & 1.528356 \\
\hline 1.114238 & 0.456558 & Q02643 & Q9UBX0 & 2692 & 8820 & 0.657681 \\
\hline 1.384687 & 0.186109 & Q02643 & P51692 & 2692 & 6777 & 1.198577 \\
\hline 1.394266 & 0.17653 & Q02643 & Q9NWF9 & 2692 & 54476 & 1.217735 \\
\hline 1.513023 & 0.057773 & Q02643 & P16473 & 2692 & 7253 & 1.45525 \\
\hline 0.550847 & 1.019949 & Q02643 & Q969G2 & 2692 & 89884 & 0.469102 \\
\hline 0.58091 & 0.989886 & Q02643 & P63092 & 2692 & 2778 & 0.408976 \\
\hline 0.675434 & 0.895363 & Q02643 & Q9UBR4 & 2692 & 8022 & 0.219929 \\
\hline 0.835487 & 0.735309 & Q02643 & Q01718 & 2692 & 4158 & 0.100177 \\
\hline 0.341347 & 1.22945 & Q02643 & P41225 & 2692 & 6658 & 0.888103 \\
\hline 1.350443 & 0.220353 & Q02643 & P07202 & 2692 & 7173 & 1.130091 \\
\hline 1.324253 & 0.246543 & Q02643 & Q08499 & 2692 & 5144 & 1.07771 \\
\hline 0.909968 & 0.660829 & Q02643 & P28069 & 2692 & 5449 & 0.249139 \\
\hline 0.562154 & 1.008642 & Q02643 & P10644 & 2692 & 5573 & 0.446488 \\
\hline 0.323228 & 1.247568 & Q02643 & Q06710 & 2692 & 7849 & 0.92434 \\
\hline 1.048347 & 0.522449 & Q02643 & 075360 & 2692 & 5626 & 0.525898 \\
\hline 0.93049 & 0.640306 & Q02643 & P32243 & 2692 & 5015 & 0.290185 \\
\hline 1.015134 & 0.555662 & Q02643 & 095467 & 2692 & 2778 & 0.459472 \\
\hline 0.695151 & 0.875646 & Q02643 & 000170 & 2692 & 9049 & 0.180495 \\
\hline 1.268621 & 0.302175 & Q02643 & Q9NSE4 & 2692 & 55699 & 0.966446 \\
\hline 1.277836 & 0.29296 & Q02643 & Q96T21 & 2692 & 79048 & 0.984876 \\
\hline 0.34239 & 1.228407 & Q02643 & 060806 & 2692 & 9095 & 0.886017 \\
\hline 1.416236 & 0.15456 & Q02643 & Q5JWF2 & 2692 & 2778 & 1.261676 \\
\hline 1.114238 & 0.456558 & Q02643 & P01225 & 2692 & 2488 & 0.657681 \\
\hline 0.619764 & 0.951032 & Q02643 & Q92847 & 2692 & 2693 & 0.331268 \\
\hline 1.35591 & 0.214886 & P51692 & P01241 & 6777 & 2688 & 1.141024 \\
\hline 1.296046 & 0.27475 & P51692 & Q06187 & 6777 & 695 & 1.021296 \\
\hline 1.369698 & 0.201098 & P51692 & P22888 & 6777 & 3973 & 1.1686 \\
\hline 1.498072 & 0.072725 & P51692 & P10828 & 6777 & 7068 & 1.425347 \\
\hline 1.383255 & 0.187542 & P51692 & P10912 & 6777 & 2690 & 1.195713 \\
\hline
\end{tabular}




\begin{tabular}{|c|c|c|c|c|c|c|}
\hline 1.465641 & 0.105155 & P51692 & Q96LT9 & 6777 & 55599 & 1.360486 \\
\hline 1.396734 & 0.174062 & P51692 & Q9UBX0 & 6777 & 8820 & 1.222672 \\
\hline 1.384687 & 0.186109 & P51692 & Q02643 & 6777 & 2692 & 1.198577 \\
\hline 1.307406 & 0.263391 & P51692 & Q9NWF9 & 6777 & 54476 & 1.044015 \\
\hline 1.293748 & 0.277048 & P51692 & P16473 & 6777 & 7253 & 1.016699 \\
\hline 1.481617 & 0.089179 & P51692 & Q969G2 & 6777 & 89884 & 1.392438 \\
\hline 1.433928 & 0.136868 & P51692 & P63092 & 6777 & 2778 & 1.297061 \\
\hline 1.411833 & 0.158963 & P51692 & Q9UBR4 & 6777 & 8022 & 1.252869 \\
\hline 1.383796 & 0.187001 & P51692 & Q01718 & 6777 & 4158 & 1.196795 \\
\hline 1.426823 & 0.143973 & P51692 & P41225 & 6777 & 6658 & 1.282849 \\
\hline 1.248137 & 0.32266 & P51692 & P07202 & 6777 & 7173 & 0.925477 \\
\hline 1.335368 & 0.235428 & P51692 & Q08499 & 6777 & 5144 & 1.09994 \\
\hline 1.360309 & 0.210488 & P51692 & P28069 & 6777 & 5449 & 1.149821 \\
\hline 1.51129 & 0.059507 & P51692 & P10644 & 6777 & 5573 & 1.451783 \\
\hline 1.439319 & 0.131477 & P51692 & Q06710 & 6777 & 7849 & 1.307842 \\
\hline 1.348275 & 0.222522 & P51692 & 075360 & 6777 & 5626 & 1.125753 \\
\hline 1.347281 & 0.223516 & P51692 & P32243 & 6777 & 5015 & 1.123765 \\
\hline 1.236194 & 0.334602 & P51692 & P84996 & 6777 & 2778 & 0.901591 \\
\hline 1.454064 & 0.116733 & P51692 & 095467 & 6777 & 2778 & 1.337331 \\
\hline 1.44663 & 0.124166 & P51692 & 000170 & 6777 & 9049 & 1.322463 \\
\hline 1.335078 & 0.235718 & P51692 & Q9NSE4 & 6777 & 55699 & 1.09936 \\
\hline 1.287642 & 0.283155 & P51692 & Q96T21 & 6777 & 79048 & 1.004487 \\
\hline 1.518076 & 0.05272 & P51692 & 060806 & 6777 & 9095 & 1.465356 \\
\hline 1.427745 & 0.143052 & P51692 & Q96P66 & 6777 & 83550 & 1.284693 \\
\hline 1.370419 & 0.200378 & P51692 & Q5JWF2 & 6777 & 2778 & 1.170041 \\
\hline 1.407656 & 0.16314 & P51692 & P01225 & 6777 & 2488 & 1.244516 \\
\hline 1.264745 & 0.306052 & P51692 & Q92847 & 6777 & 2693 & 0.958693 \\
\hline 1.232911 & 0.337885 & Q9NWF9 & P01241 & 54476 & 2688 & 0.895026 \\
\hline 1.361415 & 0.209382 & Q9NWF9 & Q06187 & 54476 & 695 & 1.152033 \\
\hline 1.4176 & 0.153196 & Q9NWF9 & P22888 & 54476 & 3973 & 1.264404 \\
\hline 1.32247 & 0.248327 & Q9NWF9 & P10828 & 54476 & 7068 & 1.074143 \\
\hline 1.368187 & 0.202609 & Q9NWF9 & P10912 & 54476 & 2690 & 1.165579 \\
\hline 1.346372 & 0.224424 & Q9NWF9 & Q96LT9 & 54476 & 55599 & 1.121948 \\
\hline 1.299569 & 0.271227 & Q9NWF9 & Q9UBX0 & 54476 & 8820 & 1.028342 \\
\hline 1.394266 & 0.17653 & Q9NWF9 & Q02643 & 54476 & 2692 & 1.217735 \\
\hline 1.307406 & 0.263391 & Q9NWF9 & P51692 & 54476 & 6777 & 1.044015 \\
\hline 1.248925 & 0.321871 & Q9NWF9 & P16473 & 54476 & 7253 & 0.927054 \\
\hline 1.350908 & 0.219889 & Q9NWF9 & Q969G2 & 54476 & 89884 & 1.131019 \\
\hline 1.404705 & 0.166092 & Q9NWF9 & P63092 & 54476 & 2778 & 1.238613 \\
\hline 1.426737 & 0.14406 & Q9NWF9 & Q9UBR4 & 54476 & 8022 & 1.282677 \\
\hline 1.40874 & 0.162057 & Q9NWF9 & Q01718 & 54476 & 4158 & 1.246683 \\
\hline
\end{tabular}




\begin{tabular}{|c|c|c|c|c|c|c|}
\hline 1.318848 & 0.251949 & Q9NWF9 & P41225 & 54476 & 6658 & 1.066899 \\
\hline 1.202469 & 0.368328 & Q9NWF9 & P07202 & 54476 & 7173 & 0.834141 \\
\hline 1.24339 & 0.327407 & Q9NWF9 & Q08499 & 54476 & 5144 & 0.915983 \\
\hline 1.310833 & 0.259963 & Q9NWF9 & P28069 & 54476 & 5449 & 1.050869 \\
\hline 1.086775 & 0.484022 & Q9NWF9 & P10644 & 54476 & 5573 & 0.602753 \\
\hline 1.388715 & 0.182082 & Q9NWF9 & Q06710 & 54476 & 7849 & 1.206633 \\
\hline 1.267145 & 0.303651 & Q9NWF9 & 075360 & 54476 & 5626 & 0.963494 \\
\hline 1.15046 & 0.420336 & Q9NWF9 & P32243 & 54476 & 5015 & 0.730124 \\
\hline 1.374186 & 0.19661 & Q9NWF9 & P84996 & 54476 & 2778 & 1.177576 \\
\hline 1.342914 & 0.227883 & Q9NWF9 & 095467 & 54476 & 2778 & 1.115031 \\
\hline 1.369729 & 0.201067 & Q9NWF9 & 000170 & 54476 & 9049 & 1.168661 \\
\hline 1.268226 & 0.30257 & Q9NWF9 & Q9NSE4 & 54476 & 55699 & 0.965657 \\
\hline 1.386591 & 0.184205 & Q9NWF9 & Q96T21 & 54476 & 79048 & 1.202386 \\
\hline 1.442912 & 0.127884 & Q9NWF9 & 060806 & 54476 & 9095 & 1.315028 \\
\hline 1.362986 & 0.20781 & Q9NWF9 & Q96P66 & 54476 & 83550 & 1.155176 \\
\hline 1.322354 & 0.248443 & Q9NWF9 & Q5JWF2 & 54476 & 2778 & 1.073911 \\
\hline 1.288588 & 0.282209 & Q9NWF9 & P01225 & 54476 & 2488 & 1.006379 \\
\hline 1.29724 & 0.273556 & Q9NWF9 & Q92847 & 54476 & 2693 & 1.023684 \\
\hline 1.300964 & 0.269832 & P16473 & P01241 & 7253 & 2688 & 1.031132 \\
\hline 1.404128 & 0.166669 & P16473 & Q06187 & 7253 & 695 & 1.237459 \\
\hline 1.420601 & 0.150195 & P16473 & P22888 & 7253 & 3973 & 1.270406 \\
\hline 1.459509 & 0.111287 & P16473 & P10828 & 7253 & 7068 & 1.348222 \\
\hline 1.25292 & 0.317876 & P16473 & P10912 & 7253 & 2690 & 0.935045 \\
\hline 1.375672 & 0.195124 & P16473 & Q96LT9 & 7253 & 55599 & 1.180548 \\
\hline 1.444509 & 0.126287 & P16473 & Q9UBX0 & 7253 & 8820 & 1.318222 \\
\hline 1.513023 & 0.057773 & P16473 & Q02643 & 7253 & 2692 & 1.45525 \\
\hline 1.293748 & 0.277048 & P16473 & P51692 & 7253 & 6777 & 1.016699 \\
\hline 1.248925 & 0.321871 & P16473 & Q9NWF9 & 7253 & 54476 & 0.927054 \\
\hline 1.482986 & 0.08781 & P16473 & Q969G2 & 7253 & 89884 & 1.395176 \\
\hline 1.339251 & 0.231545 & P16473 & P63092 & 7253 & 2778 & 1.107706 \\
\hline 1.43281 & 0.137986 & P16473 & Q9UBR4 & 7253 & 8022 & 1.294823 \\
\hline 1.376277 & 0.194519 & P16473 & Q01718 & 7253 & 4158 & 1.181758 \\
\hline 1.399421 & 0.171376 & P16473 & P41225 & 7253 & 6658 & 1.228045 \\
\hline 1.250884 & 0.319913 & P16473 & P07202 & 7253 & 7173 & 0.930971 \\
\hline 1.249884 & 0.320913 & P16473 & Q08499 & 7253 & 5144 & 0.928971 \\
\hline 1.341224 & 0.229572 & P16473 & P28069 & 7253 & 5449 & 1.111651 \\
\hline 1.37665 & 0.194146 & P16473 & P10644 & 7253 & 5573 & 1.182505 \\
\hline 1.409073 & 0.161723 & P16473 & Q06710 & 7253 & 7849 & 1.24735 \\
\hline 1.44642 & 0.124376 & P16473 & 075360 & 7253 & 5626 & 1.322044 \\
\hline 1.393419 & 0.177377 & P16473 & P32243 & 7253 & 5015 & 1.216041 \\
\hline 1.412979 & 0.157817 & P16473 & P84996 & 7253 & 2778 & 1.255162 \\
\hline
\end{tabular}




\begin{tabular}{|c|c|c|c|c|c|c|}
\hline 1.362069 & 0.208728 & P16473 & 095467 & 7253 & 2778 & 1.153341 \\
\hline 1.463919 & 0.106877 & P16473 & 000170 & 7253 & 9049 & 1.357042 \\
\hline 1.298326 & 0.272471 & P16473 & Q9NSE4 & 7253 & 55699 & 1.025855 \\
\hline 1.274305 & 0.296492 & P16473 & Q96T21 & 7253 & 79048 & 0.977813 \\
\hline 1.383992 & 0.186804 & P16473 & 060806 & 7253 & 9095 & 1.197188 \\
\hline 1.315581 & 0.255216 & P16473 & Q96P66 & 7253 & 83550 & 1.060365 \\
\hline 1.330701 & 0.240096 & P16473 & Q5JWF2 & 7253 & 2778 & 1.090605 \\
\hline 1.433063 & 0.137734 & P16473 & P01225 & 7253 & 2488 & 1.295329 \\
\hline 1.483427 & 0.08737 & P16473 & Q92847 & 7253 & 2693 & 1.396057 \\
\hline 1.060278 & 0.510519 & Q969G2 & P01241 & 89884 & 2688 & 0.549759 \\
\hline 1.447404 & 0.123393 & Q969G2 & P22888 & 89884 & 3973 & 1.324011 \\
\hline 0.288807 & 1.281989 & Q969G2 & P10828 & 89884 & 7068 & 0.993182 \\
\hline 1.437197 & 0.133599 & Q969G2 & P10912 & 89884 & 2690 & 1.303599 \\
\hline 1.152866 & 0.41793 & Q969G2 & Q9UBX0 & 89884 & 8820 & 0.734935 \\
\hline 0.550847 & 1.019949 & Q969G2 & Q02643 & 89884 & 2692 & 0.469102 \\
\hline 1.481617 & 0.089179 & Q969G2 & P51692 & 89884 & 6777 & 1.392438 \\
\hline 1.350908 & 0.219889 & Q969G2 & Q9NWF9 & 89884 & 54476 & 1.131019 \\
\hline 1.482986 & 0.08781 & Q969G2 & P16473 & 89884 & 7253 & 1.395176 \\
\hline 0.599694 & 0.971102 & Q969G2 & P63092 & 89884 & 2778 & 0.371409 \\
\hline 0.914923 & 0.655873 & Q969G2 & Q9UBR4 & 89884 & 8022 & 0.25905 \\
\hline 0.991171 & 0.579625 & Q969G2 & Q01718 & 89884 & 4158 & 0.411545 \\
\hline 0.71947 & 0.851327 & Q969G2 & P41225 & 89884 & 6658 & 0.131857 \\
\hline 1.257159 & 0.313637 & Q969G2 & P07202 & 89884 & 7173 & 0.943521 \\
\hline 1.450515 & 0.120282 & Q969G2 & Q08499 & 89884 & 5144 & 1.330233 \\
\hline 0.949388 & 0.621408 & Q969G2 & P28069 & 89884 & 5449 & 0.32798 \\
\hline 0.799678 & 0.771118 & Q969G2 & P10644 & 89884 & 5573 & 0.02856 \\
\hline 0.567262 & 1.003534 & Q969G2 & Q06710 & 89884 & 7849 & 0.436272 \\
\hline 1.070709 & 0.500087 & Q969G2 & 075360 & 89884 & 5626 & 0.570622 \\
\hline 1.03159 & 0.539206 & Q969G2 & P32243 & 89884 & 5015 & 0.492384 \\
\hline 1.096186 & 0.47461 & Q969G2 & 095467 & 89884 & 2778 & 0.621576 \\
\hline 0.841582 & 0.729215 & Q969G2 & 000170 & 89884 & 9049 & 0.112367 \\
\hline 1.213473 & 0.357323 & Q969G2 & Q9NSE4 & 89884 & 55699 & 0.85615 \\
\hline 1.453064 & 0.117732 & Q969G2 & Q96T21 & 89884 & 79048 & 1.335332 \\
\hline 0.72843 & 0.842366 & Q969G2 & 060806 & 89884 & 9095 & 0.113937 \\
\hline 1.29766 & 0.273136 & Q969G2 & Q5JWF2 & 89884 & 2778 & 1.024525 \\
\hline 1.146281 & 0.424516 & Q969G2 & P01225 & 89884 & 2488 & 0.721765 \\
\hline 0.778954 & 0.791843 & Q969G2 & Q92847 & 89884 & 2693 & 0.012889 \\
\hline 1.107344 & 0.463453 & P63092 & P01241 & 2778 & 2688 & 0.643891 \\
\hline 1.418896 & 0.1519 & P63092 & Q06187 & 2778 & 695 & 1.266995 \\
\hline 0.222237 & 1.348559 & P63092 & P10828 & 2778 & 7068 & 1.126322 \\
\hline 1.506426 & 0.06437 & P63092 & P10912 & 2778 & 2690 & 1.442056 \\
\hline
\end{tabular}




\begin{tabular}{|c|c|c|c|c|c|c|}
\hline 1.083316 & 0.48748 & P63092 & Q9UBX0 & 2778 & 8820 & 0.595836 \\
\hline 0.58091 & 0.989886 & P63092 & Q02643 & 2778 & 2692 & 0.408976 \\
\hline 1.433928 & 0.136868 & P63092 & P51692 & 2778 & 6777 & 1.297061 \\
\hline 1.404705 & 0.166092 & P63092 & Q9NWF9 & 2778 & 54476 & 1.238613 \\
\hline 1.339251 & 0.231545 & P63092 & P16473 & 2778 & 7253 & 1.107706 \\
\hline 0.599694 & 0.971102 & P63092 & Q969G2 & 2778 & 89884 & 0.371409 \\
\hline 0.754706 & 0.816091 & P63092 & Q9UBR4 & 2778 & 8022 & 0.061385 \\
\hline 0.913011 & 0.657786 & P63092 & Q01718 & 2778 & 4158 & 0.255225 \\
\hline 0.435493 & 1.135304 & P63092 & P41225 & 2778 & 6658 & 0.699811 \\
\hline 1.306075 & 0.264721 & P63092 & P07202 & 2778 & 7173 & 1.041354 \\
\hline 1.260101 & 0.310695 & P63092 & Q08499 & 2778 & 5144 & 0.949406 \\
\hline 0.866994 & 0.703802 & P63092 & P28069 & 2778 & 5449 & 0.163192 \\
\hline 0.755109 & 0.815687 & P63092 & P10644 & 2778 & 5573 & 0.060578 \\
\hline 0.552386 & 1.01841 & P63092 & Q06710 & 2778 & 7849 & 0.466024 \\
\hline 1.053037 & 0.517759 & P63092 & 075360 & 2778 & 5626 & 0.535278 \\
\hline 0.876218 & 0.694578 & P63092 & P32243 & 2778 & 5015 & 0.181641 \\
\hline 1.017381 & 0.553415 & P63092 & 095467 & 2778 & 2778 & 0.463966 \\
\hline 0.743663 & 0.827133 & P63092 & 000170 & 2778 & 9049 & 0.08347 \\
\hline 1.409249 & 0.161547 & P63092 & Q9NSE4 & 2778 & 55699 & 1.247702 \\
\hline 1.427783 & 0.143014 & P63092 & Q96T21 & 2778 & 79048 & 1.284769 \\
\hline 0.245777 & 1.325019 & P63092 & 060806 & 2778 & 9095 & 1.079242 \\
\hline 1.309296 & 0.2615 & P63092 & Q5JWF2 & 2778 & 2778 & 1.047797 \\
\hline 1.063493 & 0.507303 & P63092 & P01225 & 2778 & 2488 & 0.55619 \\
\hline 0.78674 & 0.784057 & P63092 & Q92847 & 2778 & 2693 & 0.002683 \\
\hline 1.249639 & 0.321157 & Q9UBR4 & P01241 & 8022 & 2688 & 0.928482 \\
\hline 1.542638 & 0.028159 & Q9UBR4 & Q06187 & 8022 & 695 & 1.514479 \\
\hline 1.537469 & 0.033327 & Q9UBR4 & P22888 & 8022 & 3973 & 1.504141 \\
\hline 0.449477 & 1.12132 & Q9UBR4 & P10828 & 8022 & 7068 & 0.671843 \\
\hline 1.320472 & 0.250324 & Q9UBR4 & Q9UBX0 & 8022 & 8820 & 1.070149 \\
\hline 0.675434 & 0.895363 & Q9UBR4 & Q02643 & 8022 & 2692 & 0.219929 \\
\hline 1.411833 & 0.158963 & Q9UBR4 & P51692 & 8022 & 6777 & 1.252869 \\
\hline 1.426737 & 0.14406 & Q9UBR4 & Q9NWF9 & 8022 & 54476 & 1.282677 \\
\hline 1.43281 & 0.137986 & Q9UBR4 & P16473 & 8022 & 7253 & 1.294823 \\
\hline 0.914923 & 0.655873 & Q9UBR4 & Q969G2 & 8022 & 89884 & 0.25905 \\
\hline 0.754706 & 0.816091 & Q9UBR4 & P63092 & 8022 & 2778 & 0.061385 \\
\hline 0.989862 & 0.580934 & Q9UBR4 & Q01718 & 8022 & 4158 & 0.408928 \\
\hline 0.925398 & 0.645398 & Q9UBR4 & P41225 & 8022 & 6658 & 0.280001 \\
\hline 1.307778 & 0.263018 & Q9UBR4 & P07202 & 8022 & 7173 & 1.044761 \\
\hline 1.33687 & 0.233927 & Q9UBR4 & Q08499 & 8022 & 5144 & 1.102943 \\
\hline 1.025694 & 0.545102 & Q9UBR4 & P28069 & 8022 & 5449 & 0.480592 \\
\hline 0.934738 & 0.636058 & Q9UBR4 & P10644 & 8022 & 5573 & 0.298681 \\
\hline
\end{tabular}




\begin{tabular}{|c|c|c|c|c|c|c|}
\hline 0.822657 & 0.748139 & Q9UBR4 & Q06710 & 8022 & 7849 & 0.074517 \\
\hline 1.283496 & 0.287301 & Q9UBR4 & 075360 & 8022 & 5626 & 0.996195 \\
\hline 1.115902 & 0.454894 & Q9UBR4 & P32243 & 8022 & 5015 & 0.661008 \\
\hline 0.344423 & 1.226374 & Q9UBR4 & P84996 & 8022 & 2778 & 0.881951 \\
\hline 1.286618 & 0.284178 & Q9UBR4 & 095467 & 8022 & 2778 & 1.002439 \\
\hline 0.967121 & 0.603675 & Q9UBR4 & 000170 & 8022 & 9049 & 0.363446 \\
\hline 1.272805 & 0.297992 & Q9UBR4 & Q9NSE4 & 8022 & 55699 & 0.974813 \\
\hline 1.409325 & 0.161471 & Q9UBR4 & Q96T21 & 8022 & 79048 & 1.247853 \\
\hline 0.520566 & 1.05023 & Q9UBR4 & 060806 & 8022 & 9095 & 0.529664 \\
\hline 1.245408 & 0.325388 & Q9UBR4 & Q5JWF2 & 8022 & 2778 & 0.92002 \\
\hline 1.320472 & 0.250324 & Q9UBR4 & P01225 & 8022 & 2488 & 1.070149 \\
\hline 0.823594 & 0.747202 & Q9UBR4 & Q92847 & 8022 & 2693 & 0.076391 \\
\hline 1.478867 & 0.091929 & Q01718 & P01241 & 4158 & 2688 & 1.386938 \\
\hline 1.470784 & 0.100012 & Q01718 & Q06187 & 4158 & 695 & 1.370772 \\
\hline 1.440933 & 0.129863 & Q01718 & P22888 & 4158 & 3973 & 1.31107 \\
\hline 0.774031 & 0.796766 & Q01718 & P10828 & 4158 & 7068 & 0.022735 \\
\hline 1.370689 & 0.200108 & Q01718 & P10912 & 4158 & 2690 & 1.170581 \\
\hline 1.519538 & 0.051258 & Q01718 & Q9UBX0 & 4158 & 8820 & 1.46828 \\
\hline 0.835487 & 0.735309 & Q01718 & Q02643 & 4158 & 2692 & 0.100177 \\
\hline 1.383796 & 0.187001 & Q01718 & P51692 & 4158 & 6777 & 1.196795 \\
\hline 1.40874 & 0.162057 & Q01718 & Q9NWF9 & 4158 & 54476 & 1.246683 \\
\hline 1.376277 & 0.194519 & Q01718 & P16473 & 4158 & 7253 & 1.181758 \\
\hline 0.991171 & 0.579625 & Q01718 & Q969G2 & 4158 & 89884 & 0.411545 \\
\hline 0.913011 & 0.657786 & Q01718 & P63092 & 4158 & 2778 & 0.255225 \\
\hline 0.989862 & 0.580934 & Q01718 & Q9UBR4 & 4158 & 8022 & 0.408928 \\
\hline 0.883619 & 0.687178 & Q01718 & P41225 & 4158 & 6658 & 0.196441 \\
\hline 1.346074 & 0.224722 & Q01718 & P07202 & 4158 & 7173 & 1.121352 \\
\hline 1.361793 & 0.209003 & Q01718 & Q08499 & 4158 & 5144 & 1.15279 \\
\hline 1.336378 & 0.234419 & Q01718 & P28069 & 4158 & 5449 & 1.101959 \\
\hline 1.100489 & 0.470308 & Q01718 & P10644 & 4158 & 5573 & 0.630181 \\
\hline 1.011722 & 0.559074 & Q01718 & Q06710 & 4158 & 7849 & 0.452648 \\
\hline 1.501535 & 0.069261 & Q01718 & 075360 & 4158 & 5626 & 1.432274 \\
\hline 1.3739 & 0.196896 & Q01718 & P32243 & 4158 & 5015 & 1.177004 \\
\hline 1.43207 & 0.138727 & Q01718 & 095467 & 4158 & 2778 & 1.293343 \\
\hline 1.133558 & 0.437238 & Q01718 & 000170 & 4158 & 9049 & 0.69632 \\
\hline 1.224031 & 0.346765 & Q01718 & Q9NSE4 & 4158 & 55699 & 0.877265 \\
\hline 1.23534 & 0.335457 & Q01718 & Q96T21 & 4158 & 79048 & 0.899883 \\
\hline 1.029536 & 0.54126 & Q01718 & 060806 & 4158 & 9095 & 0.488276 \\
\hline 0.018742 & 1.552055 & Q01718 & Q96P66 & 4158 & 83550 & 1.533313 \\
\hline 1.229742 & 0.341054 & Q01718 & Q5JWF2 & 4158 & 2778 & 0.888688 \\
\hline 1.524662 & 0.046135 & Q01718 & P01225 & 4158 & 2488 & 1.478527 \\
\hline
\end{tabular}




\begin{tabular}{|c|c|c|c|c|c|c|}
\hline 1.079356 & 0.49144 & Q01718 & Q92847 & 4158 & 2693 & 0.587916 \\
\hline 1.090885 & 0.479912 & P41225 & P01241 & 6658 & 2688 & 0.610973 \\
\hline 1.239331 & 0.331465 & P41225 & P10912 & 6658 & 2690 & 0.907866 \\
\hline 1.167718 & 0.403078 & P41225 & Q9UBX0 & 6658 & 8820 & 0.76464 \\
\hline 0.341347 & 1.22945 & P41225 & Q02643 & 6658 & 2692 & 0.888103 \\
\hline 1.426823 & 0.143973 & P41225 & P51692 & 6658 & 6777 & 1.282849 \\
\hline 1.318848 & 0.251949 & P41225 & Q9NWF9 & 6658 & 54476 & 1.066899 \\
\hline 1.399421 & 0.171376 & P41225 & P16473 & 6658 & 7253 & 1.228045 \\
\hline 0.71947 & 0.851327 & P41225 & Q969G2 & 6658 & 89884 & 0.131857 \\
\hline 0.435493 & 1.135304 & P41225 & P63092 & 6658 & 2778 & 0.699811 \\
\hline 0.925398 & 0.645398 & P41225 & Q9UBR4 & 6658 & 8022 & 0.280001 \\
\hline 0.883619 & 0.687178 & P41225 & Q01718 & 6658 & 4158 & 0.196441 \\
\hline 1.192374 & 0.378422 & P41225 & P07202 & 6658 & 7173 & 0.813952 \\
\hline 1.37574 & 0.195056 & P41225 & Q08499 & 6658 & 5144 & 1.180685 \\
\hline 0.938106 & 0.63269 & P41225 & P28069 & 6658 & 5449 & 0.305415 \\
\hline 0.5614 & 1.009397 & P41225 & P10644 & 6658 & 5573 & 0.447997 \\
\hline 0.601149 & 0.969647 & P41225 & Q06710 & 6658 & 7849 & 0.368498 \\
\hline 1.191901 & 0.378896 & P41225 & 075360 & 6658 & 5626 & 0.813005 \\
\hline 1.057953 & 0.512843 & P41225 & P32243 & 6658 & 5015 & 0.545111 \\
\hline 1.479301 & 0.091495 & P41225 & P84996 & 6658 & 2778 & 1.387806 \\
\hline 1.024845 & 0.545952 & P41225 & 095467 & 6658 & 2778 & 0.478893 \\
\hline 0.799039 & 0.771757 & P41225 & 000170 & 6658 & 9049 & 0.027282 \\
\hline 1.299074 & 0.271722 & P41225 & Q9NSE4 & 6658 & 55699 & 1.027352 \\
\hline 1.232987 & 0.33781 & P41225 & Q96T21 & 6658 & 79048 & 0.895177 \\
\hline 0.541078 & 1.029718 & P41225 & 060806 & 6658 & 9095 & 0.48864 \\
\hline 1.302927 & 0.267869 & P41225 & Q5JWF2 & 6658 & 2778 & 1.035058 \\
\hline 1.160764 & 0.410032 & P41225 & P01225 & 6658 & 2488 & 0.750732 \\
\hline 0.617378 & 0.953418 & P41225 & Q92847 & 6658 & 2693 & 0.336041 \\
\hline 1.338789 & 0.232007 & P07202 & P01241 & 7173 & 2688 & 1.106782 \\
\hline 1.276269 & 0.294527 & P07202 & Q06187 & 7173 & 695 & 0.981742 \\
\hline 1.307605 & 0.263191 & P07202 & P22888 & 7173 & 3973 & 1.044414 \\
\hline 1.262976 & 0.30782 & P07202 & P10828 & 7173 & 7068 & 0.955156 \\
\hline 1.361993 & 0.208803 & P07202 & P10912 & 7173 & 2690 & 1.15319 \\
\hline 1.362634 & 0.208162 & P07202 & Q96LT9 & 7173 & 55599 & 1.154473 \\
\hline 1.323535 & 0.247261 & P07202 & Q9UBX0 & 7173 & 8820 & 1.076274 \\
\hline 1.350443 & 0.220353 & P07202 & Q02643 & 7173 & 2692 & 1.130091 \\
\hline 1.248137 & 0.32266 & P07202 & P51692 & 7173 & 6777 & 0.925477 \\
\hline 1.202469 & 0.368328 & P07202 & Q9NWF9 & 7173 & 54476 & 0.834141 \\
\hline 1.250884 & 0.319913 & P07202 & P16473 & 7173 & 7253 & 0.930971 \\
\hline 1.257159 & 0.313637 & P07202 & Q969G2 & 7173 & 89884 & 0.943521 \\
\hline 1.306075 & 0.264721 & P07202 & P63092 & 7173 & 2778 & 1.041354 \\
\hline
\end{tabular}




\begin{tabular}{|c|c|c|c|c|c|c|}
\hline 1.307778 & 0.263018 & P07202 & Q9UBR4 & 7173 & 8022 & 1.044761 \\
\hline 1.346074 & 0.224722 & P07202 & Q01718 & 7173 & 4158 & 1.121352 \\
\hline 1.192374 & 0.378422 & P07202 & P41225 & 7173 & 6658 & 0.813952 \\
\hline 1.384651 & 0.186146 & P07202 & Q08499 & 7173 & 5144 & 1.198505 \\
\hline 1.293259 & 0.277538 & P07202 & P28069 & 7173 & 5449 & 1.015721 \\
\hline 1.283967 & 0.286829 & P07202 & P10644 & 7173 & 5573 & 0.997138 \\
\hline 1.292017 & 0.278779 & P07202 & Q06710 & 7173 & 7849 & 1.013238 \\
\hline 1.286176 & 0.28462 & P07202 & 075360 & 7173 & 5626 & 1.001556 \\
\hline 1.267496 & 0.3033 & P07202 & P32243 & 7173 & 5015 & 0.964196 \\
\hline 1.238581 & 0.332215 & P07202 & P84996 & 7173 & 2778 & 0.906366 \\
\hline 1.30601 & 0.264786 & P07202 & 095467 & 7173 & 2778 & 1.041224 \\
\hline 1.274641 & 0.296155 & P07202 & 000170 & 7173 & 9049 & 0.978486 \\
\hline 1.280999 & 0.289797 & P07202 & Q9NSE4 & 7173 & 55699 & 0.991202 \\
\hline 1.138106 & 0.43269 & P07202 & Q96T21 & 7173 & 79048 & 0.705416 \\
\hline 1.2119 & 0.358897 & P07202 & 060806 & 7173 & 9095 & 0.853003 \\
\hline 1.298415 & 0.272381 & P07202 & Q96P66 & 7173 & 83550 & 1.026033 \\
\hline 1.224617 & 0.346179 & P07202 & Q5JWF2 & 7173 & 2778 & 0.878438 \\
\hline 1.332645 & 0.238151 & P07202 & P01225 & 7173 & 2488 & 1.094494 \\
\hline 1.295971 & 0.274825 & P07202 & Q92847 & 7173 & 2693 & 1.021146 \\
\hline 1.273813 & 0.296983 & Q08499 & P01241 & 5144 & 2688 & 0.97683 \\
\hline 1.242438 & 0.328359 & Q08499 & Q06187 & 5144 & 695 & 0.914079 \\
\hline 1.320309 & 0.250487 & Q08499 & P22888 & 5144 & 3973 & 1.069822 \\
\hline 1.398481 & 0.172315 & Q08499 & P10828 & 5144 & 7068 & 1.226166 \\
\hline 1.46161 & 0.109187 & Q08499 & P10912 & 5144 & 2690 & 1.352423 \\
\hline 1.344559 & 0.226238 & Q08499 & Q96LT9 & 5144 & 55599 & 1.118321 \\
\hline 1.311002 & 0.259794 & Q08499 & Q9UBX0 & 5144 & 8820 & 1.051207 \\
\hline 1.324253 & 0.246543 & Q08499 & Q02643 & 5144 & 2692 & 1.07771 \\
\hline 1.335368 & 0.235428 & Q08499 & P51692 & 5144 & 6777 & 1.09994 \\
\hline 1.24339 & 0.327407 & Q08499 & Q9NWF9 & 5144 & 54476 & 0.915983 \\
\hline 1.249884 & 0.320913 & Q08499 & P16473 & 5144 & 7253 & 0.928971 \\
\hline 1.450515 & 0.120282 & Q08499 & Q969G2 & 5144 & 89884 & 1.330233 \\
\hline 1.260101 & 0.310695 & Q08499 & P63092 & 5144 & 2778 & 0.949406 \\
\hline 1.33687 & 0.233927 & Q08499 & Q9UBR4 & 5144 & 8022 & 1.102943 \\
\hline 1.361793 & 0.209003 & Q08499 & Q01718 & 5144 & 4158 & 1.15279 \\
\hline 1.37574 & 0.195056 & Q08499 & P41225 & 5144 & 6658 & 1.180685 \\
\hline 1.384651 & 0.186146 & Q08499 & P07202 & 5144 & 7173 & 1.198505 \\
\hline 1.333114 & 0.237683 & Q08499 & P28069 & 5144 & 5449 & 1.095431 \\
\hline 1.180279 & 0.390517 & Q08499 & P10644 & 5144 & 5573 & 0.789761 \\
\hline 1.425399 & 0.145397 & Q08499 & Q06710 & 5144 & 7849 & 1.280003 \\
\hline 1.31381 & 0.256987 & Q08499 & 075360 & 5144 & 5626 & 1.056823 \\
\hline 1.417162 & 0.153635 & Q08499 & P32243 & 5144 & 5015 & 1.263527 \\
\hline
\end{tabular}




\begin{tabular}{|c|c|c|c|c|c|c|}
\hline 1.260009 & 0.310788 & Q08499 & P84996 & 5144 & 2778 & 0.949221 \\
\hline 1.291273 & 0.279523 & Q08499 & 095467 & 5144 & 2778 & 1.01175 \\
\hline 1.486163 & 0.084633 & Q08499 & 000170 & 5144 & 9049 & 1.40153 \\
\hline 1.296319 & 0.274477 & Q08499 & Q9NSE4 & 5144 & 55699 & 1.021842 \\
\hline 1.240415 & 0.330382 & Q08499 & Q96T21 & 5144 & 79048 & 0.910033 \\
\hline 1.457692 & 0.113104 & Q08499 & 060806 & 5144 & 9095 & 1.344588 \\
\hline 1.345676 & 0.22512 & Q08499 & Q96P66 & 5144 & 83550 & 1.120556 \\
\hline 1.307771 & 0.263025 & Q08499 & Q5JWF2 & 5144 & 2778 & 1.044746 \\
\hline 1.299307 & 0.271489 & Q08499 & P01225 & 5144 & 2488 & 1.027818 \\
\hline 1.321346 & 0.24945 & Q08499 & Q92847 & 5144 & 2693 & 1.071896 \\
\hline 1.492543 & 0.078254 & P28069 & P01241 & 5449 & 2688 & 1.414289 \\
\hline 1.42306 & 0.147736 & P28069 & Q06187 & 5449 & 695 & 1.275324 \\
\hline 1.453773 & 0.117024 & P28069 & P22888 & 5449 & 3973 & 1.336749 \\
\hline 0.795252 & 0.775545 & P28069 & P10828 & 5449 & 7068 & 0.019707 \\
\hline 1.539218 & 0.031579 & P28069 & P10912 & 5449 & 2690 & 1.507639 \\
\hline 1.521566 & 0.04923 & P28069 & Q9UBX0 & 5449 & 8820 & 1.472336 \\
\hline 0.909968 & 0.660829 & P28069 & Q02643 & 5449 & 2692 & 0.249139 \\
\hline 1.360309 & 0.210488 & P28069 & P51692 & 5449 & 6777 & 1.149821 \\
\hline 1.310833 & 0.259963 & P28069 & Q9NWF9 & 5449 & 54476 & 1.050869 \\
\hline 1.341224 & 0.229572 & P28069 & P16473 & 5449 & 7253 & 1.111651 \\
\hline 0.949388 & 0.621408 & P28069 & Q969G2 & 5449 & 89884 & 0.32798 \\
\hline 0.866994 & 0.703802 & P28069 & P63092 & 5449 & 2778 & 0.163192 \\
\hline 1.025694 & 0.545102 & P28069 & Q9UBR4 & 5449 & 8022 & 0.480592 \\
\hline 1.336378 & 0.234419 & P28069 & Q01718 & 5449 & 4158 & 1.101959 \\
\hline 0.938106 & 0.63269 & P28069 & P41225 & 5449 & 6658 & 0.305415 \\
\hline 1.293259 & 0.277538 & P28069 & P07202 & 5449 & 7173 & 1.015721 \\
\hline 1.333114 & 0.237683 & P28069 & Q08499 & 5449 & 5144 & 1.095431 \\
\hline 1.114635 & 0.456161 & P28069 & P10644 & 5449 & 5573 & 0.658474 \\
\hline 0.879032 & 0.691765 & P28069 & Q06710 & 5449 & 7849 & 0.187267 \\
\hline 1.458223 & 0.112573 & P28069 & 075360 & 5449 & 5626 & 1.345649 \\
\hline 1.353623 & 0.217173 & P28069 & P32243 & 5449 & 5015 & 1.13645 \\
\hline 1.204363 & 0.366433 & P28069 & P84996 & 5449 & 2778 & 0.83793 \\
\hline 1.485822 & 0.084974 & P28069 & 095467 & 5449 & 2778 & 1.400848 \\
\hline 1.161033 & 0.409763 & P28069 & 000170 & 5449 & 9049 & 0.751269 \\
\hline 1.214772 & 0.356025 & P28069 & Q9NSE4 & 5449 & 55699 & 0.858747 \\
\hline 1.349528 & 0.221268 & P28069 & Q96T21 & 5449 & 79048 & 1.128261 \\
\hline 0.848804 & 0.721992 & P28069 & 060806 & 5449 & 9095 & 0.126812 \\
\hline 1.215962 & 0.354835 & P28069 & Q5JWF2 & 5449 & 2778 & 0.861127 \\
\hline 1.514932 & 0.055864 & P28069 & P01225 & 5449 & 2488 & 1.459068 \\
\hline 1.066575 & 0.504222 & P28069 & Q92847 & 5449 & 2693 & 0.562353 \\
\hline 1.210249 & 0.360547 & P10644 & P01241 & 5573 & 2688 & 0.849702 \\
\hline
\end{tabular}




\begin{tabular}{|c|c|c|c|c|c|c|}
\hline 1.509098 & 0.061698 & P10644 & Q06187 & 5573 & 695 & 1.4474 \\
\hline 1.46422 & 0.106576 & P10644 & P22888 & 5573 & 3973 & 1.357643 \\
\hline 0.398144 & 1.172652 & P10644 & P10828 & 5573 & 7068 & 0.774507 \\
\hline 1.434003 & 0.136793 & P10644 & P10912 & 5573 & 2690 & 1.29721 \\
\hline 1.242879 & 0.327917 & P10644 & Q9UBX0 & 5573 & 8820 & 0.914962 \\
\hline 0.562154 & 1.008642 & P10644 & Q02643 & 5573 & 2692 & 0.446488 \\
\hline 1.51129 & 0.059507 & P10644 & P51692 & 5573 & 6777 & 1.451783 \\
\hline 1.086775 & 0.484022 & P10644 & Q9NWF9 & 5573 & 54476 & 0.602753 \\
\hline 1.37665 & 0.194146 & P10644 & P16473 & 5573 & 7253 & 1.182505 \\
\hline 0.799678 & 0.771118 & P10644 & Q969G2 & 5573 & 89884 & 0.02856 \\
\hline 0.755109 & 0.815687 & P10644 & P63092 & 5573 & 2778 & 0.060578 \\
\hline 0.934738 & 0.636058 & P10644 & Q9UBR4 & 5573 & 8022 & 0.298681 \\
\hline 1.100489 & 0.470308 & P10644 & Q01718 & 5573 & 4158 & 0.630181 \\
\hline 0.5614 & 1.009397 & P10644 & P41225 & 5573 & 6658 & 0.447997 \\
\hline 1.283967 & 0.286829 & P10644 & P07202 & 5573 & 7173 & 0.997138 \\
\hline 1.180279 & 0.390517 & P10644 & Q08499 & 5573 & 5144 & 0.789761 \\
\hline 1.114635 & 0.456161 & P10644 & P28069 & 5573 & 5449 & 0.658474 \\
\hline 0.569726 & 1.00107 & P10644 & Q06710 & 5573 & 7849 & 0.431344 \\
\hline 1.244802 & 0.325994 & P10644 & 075360 & 5573 & 5626 & 0.918808 \\
\hline 1.158278 & 0.412518 & P10644 & P32243 & 5573 & 5015 & 0.74576 \\
\hline 1.185023 & 0.385773 & P10644 & 095467 & 5573 & 2778 & 0.799249 \\
\hline 0.897746 & 0.673051 & P10644 & 000170 & 5573 & 9049 & 0.224695 \\
\hline 1.08998 & 0.480816 & P10644 & Q9NSE4 & 5573 & 55699 & 0.609163 \\
\hline 1.314192 & 0.256604 & P10644 & Q96T21 & 5573 & 79048 & 1.057588 \\
\hline 0.457144 & 1.113652 & P10644 & 060806 & 5573 & 9095 & 0.656508 \\
\hline 1.181285 & 0.389511 & P10644 & Q5JWF2 & 5573 & 2778 & 0.791774 \\
\hline 1.249079 & 0.321718 & P10644 & P01225 & 5573 & 2488 & 0.927361 \\
\hline 0.884027 & 0.686769 & P10644 & Q92847 & 5573 & 2693 & 0.197259 \\
\hline 1.289006 & 0.28179 & Q06710 & P01241 & 7849 & 2688 & 1.007216 \\
\hline 1.463946 & 0.10685 & Q06710 & Q06187 & 7849 & 695 & 1.357096 \\
\hline 1.46447 & 0.106326 & Q06710 & P22888 & 7849 & 3973 & 1.358145 \\
\hline 1.450286 & 0.12051 & Q06710 & P10912 & 7849 & 2690 & 1.329776 \\
\hline 1.362044 & 0.208753 & Q06710 & Q9UBX0 & 7849 & 8820 & 1.153291 \\
\hline 0.323228 & 1.247568 & Q06710 & Q02643 & 7849 & 2692 & 0.92434 \\
\hline 1.439319 & 0.131477 & Q06710 & P51692 & 7849 & 6777 & 1.307842 \\
\hline 1.388715 & 0.182082 & Q06710 & Q9NWF9 & 7849 & 54476 & 1.206633 \\
\hline 1.409073 & 0.161723 & Q06710 & P16473 & 7849 & 7253 & 1.24735 \\
\hline 0.567262 & 1.003534 & Q06710 & Q969G2 & 7849 & 89884 & 0.436272 \\
\hline 0.552386 & 1.01841 & Q06710 & P63092 & 7849 & 2778 & 0.466024 \\
\hline 0.822657 & 0.748139 & Q06710 & Q9UBR4 & 7849 & 8022 & 0.074517 \\
\hline 1.011722 & 0.559074 & Q06710 & Q01718 & 7849 & 4158 & 0.452648 \\
\hline
\end{tabular}




\begin{tabular}{|c|c|c|c|c|c|c|}
\hline 0.601149 & 0.969647 & Q06710 & P41225 & 7849 & 6658 & 0.368498 \\
\hline 1.292017 & 0.278779 & Q06710 & P07202 & 7849 & 7173 & 1.013238 \\
\hline 1.425399 & 0.145397 & Q06710 & Q08499 & 7849 & 5144 & 1.280003 \\
\hline 0.879032 & 0.691765 & Q06710 & P28069 & 7849 & 5449 & 0.187267 \\
\hline 0.569726 & 1.00107 & Q06710 & P10644 & 7849 & 5573 & 0.431344 \\
\hline 1.321753 & 0.249043 & Q06710 & 075360 & 7849 & 5626 & 1.07271 \\
\hline 1.094416 & 0.476381 & Q06710 & P32243 & 7849 & 5015 & 0.618035 \\
\hline 1.171135 & 0.399661 & Q06710 & P84996 & 7849 & 2778 & 0.771473 \\
\hline 1.276541 & 0.294255 & Q06710 & 095467 & 7849 & 2778 & 0.982286 \\
\hline 0.969472 & 0.601324 & Q06710 & 000170 & 7849 & 9049 & 0.368148 \\
\hline 1.155209 & 0.415587 & Q06710 & Q9NSE4 & 7849 & 55699 & 0.739622 \\
\hline 1.195045 & 0.375752 & Q06710 & Q96T21 & 7849 & 79048 & 0.819293 \\
\hline 0.62742 & 0.943376 & Q06710 & 060806 & 7849 & 9095 & 0.315956 \\
\hline 1.294907 & 0.27589 & Q06710 & Q5JWF2 & 7849 & 2778 & 1.019017 \\
\hline 1.351299 & 0.219497 & Q06710 & P01225 & 7849 & 2488 & 1.131802 \\
\hline 0.556829 & 1.013968 & Q06710 & Q92847 & 7849 & 2693 & 0.457139 \\
\hline 1.450077 & 0.120719 & 075360 & Q06187 & 5626 & 695 & 1.329358 \\
\hline 1.48284 & 0.087957 & 075360 & P22888 & 5626 & 3973 & 1.394883 \\
\hline 1.087061 & 0.483735 & 075360 & P10828 & 5626 & 7068 & 0.603326 \\
\hline 1.369798 & 0.200999 & 075360 & P10912 & 5626 & 2690 & 1.168799 \\
\hline 0.010763 & 1.560033 & 075360 & Q96LT9 & 5626 & 55599 & 1.54927 \\
\hline 1.048347 & 0.522449 & 075360 & Q02643 & 5626 & 2692 & 0.525898 \\
\hline 1.348275 & 0.222522 & 075360 & P51692 & 5626 & 6777 & 1.125753 \\
\hline 1.267145 & 0.303651 & 075360 & Q9NWF9 & 5626 & 54476 & 0.963494 \\
\hline 1.44642 & 0.124376 & 075360 & P16473 & 5626 & 7253 & 1.322044 \\
\hline 1.070709 & 0.500087 & 075360 & Q969G2 & 5626 & 89884 & 0.570622 \\
\hline 1.053037 & 0.517759 & 075360 & P63092 & 5626 & 2778 & 0.535278 \\
\hline 1.283496 & 0.287301 & 075360 & Q9UBR4 & 5626 & 8022 & 0.996195 \\
\hline 1.501535 & 0.069261 & 075360 & Q01718 & 5626 & 4158 & 1.432274 \\
\hline 1.191901 & 0.378896 & 075360 & P41225 & 5626 & 6658 & 0.813005 \\
\hline 1.286176 & 0.28462 & 075360 & P07202 & 5626 & 7173 & 1.001556 \\
\hline 1.31381 & 0.256987 & 075360 & Q08499 & 5626 & 5144 & 1.056823 \\
\hline 1.458223 & 0.112573 & 075360 & P28069 & 5626 & 5449 & 1.345649 \\
\hline 1.244802 & 0.325994 & 075360 & P10644 & 5626 & 5573 & 0.918808 \\
\hline 1.321753 & 0.249043 & 075360 & Q06710 & 5626 & 7849 & 1.07271 \\
\hline 1.543303 & 0.027493 & 075360 & P32243 & 5626 & 5015 & 1.51581 \\
\hline 1.285706 & 0.28509 & 075360 & 000170 & 5626 & 9049 & 1.000616 \\
\hline 1.226141 & 0.344655 & 075360 & Q9NSE4 & 5626 & 55699 & 0.881485 \\
\hline 1.164328 & 0.406469 & 075360 & Q96T21 & 5626 & 79048 & 0.757859 \\
\hline 1.098794 & 0.472003 & 075360 & 060806 & 5626 & 9095 & 0.626791 \\
\hline 0.17642 & 1.394376 & 075360 & Q96P66 & 5626 & 83550 & 1.217957 \\
\hline
\end{tabular}




\begin{tabular}{|c|c|c|c|c|c|c|}
\hline 1.298541 & 0.272255 & 075360 & Q5JWF2 & 5626 & 2778 & 1.026286 \\
\hline 1.172249 & 0.398547 & 075360 & Q92847 & 5626 & 2693 & 0.773702 \\
\hline 1.547491 & 0.023305 & P32243 & P01241 & 5015 & 2688 & 1.524186 \\
\hline 1.530523 & 0.040273 & P32243 & Q06187 & 5015 & 695 & 1.490251 \\
\hline 0.706874 & 0.863922 & P32243 & P10828 & 5015 & 7068 & 0.157048 \\
\hline 1.552789 & 0.018007 & P32243 & P10912 & 5015 & 2690 & 1.534782 \\
\hline 0.93049 & 0.640306 & P32243 & Q02643 & 5015 & 2692 & 0.290185 \\
\hline 1.347281 & 0.223516 & P32243 & P51692 & 5015 & 6777 & 1.123765 \\
\hline 1.15046 & 0.420336 & P32243 & Q9NWF9 & 5015 & 54476 & 0.730124 \\
\hline 1.393419 & 0.177377 & P32243 & P16473 & 5015 & 7253 & 1.216041 \\
\hline 1.03159 & 0.539206 & P32243 & Q969G2 & 5015 & 89884 & 0.492384 \\
\hline 0.876218 & 0.694578 & P32243 & P63092 & 5015 & 2778 & 0.181641 \\
\hline 1.115902 & 0.454894 & P32243 & Q9UBR4 & 5015 & 8022 & 0.661008 \\
\hline 1.3739 & 0.196896 & P32243 & Q01718 & 5015 & 4158 & 1.177004 \\
\hline 1.057953 & 0.512843 & P32243 & P41225 & 5015 & 6658 & 0.545111 \\
\hline 1.267496 & 0.3033 & P32243 & P07202 & 5015 & 7173 & 0.964196 \\
\hline 1.417162 & 0.153635 & P32243 & Q08499 & 5015 & 5144 & 1.263527 \\
\hline 1.353623 & 0.217173 & P32243 & P28069 & 5015 & 5449 & 1.13645 \\
\hline 1.158278 & 0.412518 & P32243 & P10644 & 5015 & 5573 & 0.74576 \\
\hline 1.094416 & 0.476381 & P32243 & Q06710 & 5015 & 7849 & 0.618035 \\
\hline 1.543303 & 0.027493 & P32243 & 075360 & 5015 & 5626 & 1.51581 \\
\hline 1.4884 & 0.082397 & P32243 & 095467 & 5015 & 2778 & 1.406003 \\
\hline 1.116321 & 0.454475 & P32243 & 000170 & 5015 & 9049 & 0.661847 \\
\hline 1.196441 & 0.374355 & P32243 & Q9NSE4 & 5015 & 55699 & 0.822087 \\
\hline 1.196647 & 0.37415 & P32243 & Q96T21 & 5015 & 79048 & 0.822497 \\
\hline 0.831478 & 0.739318 & P32243 & 060806 & 5015 & 9095 & 0.092159 \\
\hline 1.359069 & 0.211728 & P32243 & Q5JWF2 & 5015 & 2778 & 1.147341 \\
\hline 1.026431 & 0.544365 & P32243 & Q92847 & 5015 & 2693 & 0.482067 \\
\hline 1.476387 & 0.094409 & P84996 & Q06187 & 2778 & 695 & 1.381978 \\
\hline 1.245642 & 0.325154 & P84996 & P22888 & 2778 & 3973 & 0.920488 \\
\hline 1.513465 & 0.057331 & P84996 & P10828 & 2778 & 7068 & 1.456134 \\
\hline 1.391804 & 0.178993 & P84996 & P10912 & 2778 & 2690 & 1.212811 \\
\hline 1.386692 & 0.184105 & P84996 & Q96LT9 & 2778 & 55599 & 1.202587 \\
\hline 1.236194 & 0.334602 & P84996 & P51692 & 2778 & 6777 & 0.901591 \\
\hline 1.374186 & 0.19661 & P84996 & Q9NWF9 & 2778 & 54476 & 1.177576 \\
\hline 1.412979 & 0.157817 & P84996 & P16473 & 2778 & 7253 & 1.255162 \\
\hline 0.344423 & 1.226374 & P84996 & Q9UBR4 & 2778 & 8022 & 0.881951 \\
\hline 1.479301 & 0.091495 & P84996 & P41225 & 2778 & 6658 & 1.387806 \\
\hline 1.238581 & 0.332215 & P84996 & P07202 & 2778 & 7173 & 0.906366 \\
\hline 1.260009 & 0.310788 & P84996 & Q08499 & 2778 & 5144 & 0.949221 \\
\hline 1.204363 & 0.366433 & P84996 & P28069 & 2778 & 5449 & 0.83793 \\
\hline
\end{tabular}




\begin{tabular}{|c|c|c|c|c|c|c|}
\hline 1.171135 & 0.399661 & P84996 & Q06710 & 2778 & 7849 & 0.771473 \\
\hline 1.249051 & 0.321745 & P84996 & Q9NSE4 & 2778 & 55699 & 0.927306 \\
\hline 1.320837 & 0.24996 & P84996 & Q96T21 & 2778 & 79048 & 1.070877 \\
\hline 1.111115 & 0.459681 & P84996 & Q96P66 & 2778 & 83550 & 0.651434 \\
\hline 1.420672 & 0.150124 & P84996 & Q5JWF2 & 2778 & 2778 & 1.270548 \\
\hline 0.905209 & 0.665587 & P84996 & Q92847 & 2778 & 2693 & 0.239622 \\
\hline 1.361859 & 0.208938 & 095467 & Q06187 & 2778 & 695 & 1.152921 \\
\hline 1.336192 & 0.234604 & 095467 & P22888 & 2778 & 3973 & 1.101589 \\
\hline 0.858138 & 0.712658 & 095467 & P10828 & 2778 & 7068 & 0.14548 \\
\hline 1.482129 & 0.088667 & 095467 & P10912 & 2778 & 2690 & 1.393462 \\
\hline 0.590527 & 0.980269 & 095467 & Q96LT9 & 2778 & 55599 & 0.389743 \\
\hline 1.015134 & 0.555662 & 095467 & Q02643 & 2778 & 2692 & 0.459472 \\
\hline 1.454064 & 0.116733 & 095467 & P51692 & 2778 & 6777 & 1.337331 \\
\hline 1.342914 & 0.227883 & 095467 & Q9NWF9 & 2778 & 54476 & 1.115031 \\
\hline 1.362069 & 0.208728 & 095467 & P16473 & 2778 & 7253 & 1.153341 \\
\hline 1.096186 & 0.47461 & 095467 & Q969G2 & 2778 & 89884 & 0.621576 \\
\hline 1.017381 & 0.553415 & 095467 & P63092 & 2778 & 2778 & 0.463966 \\
\hline 1.286618 & 0.284178 & 095467 & Q9UBR4 & 2778 & 8022 & 1.002439 \\
\hline 1.43207 & 0.138727 & 095467 & Q01718 & 2778 & 4158 & 1.293343 \\
\hline 1.024845 & 0.545952 & 095467 & P41225 & 2778 & 6658 & 0.478893 \\
\hline 1.30601 & 0.264786 & 095467 & P07202 & 2778 & 7173 & 1.041224 \\
\hline 1.291273 & 0.279523 & 095467 & Q08499 & 2778 & 5144 & 1.01175 \\
\hline 1.485822 & 0.084974 & 095467 & P28069 & 2778 & 5449 & 1.400848 \\
\hline 1.185023 & 0.385773 & 095467 & P10644 & 2778 & 5573 & 0.799249 \\
\hline 1.276541 & 0.294255 & 095467 & Q06710 & 2778 & 7849 & 0.982286 \\
\hline 1.4884 & 0.082397 & 095467 & P32243 & 2778 & 5015 & 1.406003 \\
\hline 1.256809 & 0.313987 & 095467 & 000170 & 2778 & 9049 & 0.942821 \\
\hline 1.220246 & 0.350551 & 095467 & Q9NSE4 & 2778 & 55699 & 0.869695 \\
\hline 1.208142 & 0.362655 & 095467 & Q96T21 & 2778 & 79048 & 0.845487 \\
\hline 0.964666 & 0.60613 & 095467 & 060806 & 2778 & 9095 & 0.358537 \\
\hline 0.333373 & 1.237423 & 095467 & Q96P66 & 2778 & 83550 & 0.904049 \\
\hline 1.211925 & 0.358872 & 095467 & Q5JWF2 & 2778 & 2778 & 0.853053 \\
\hline 1.190344 & 0.380452 & 095467 & Q92847 & 2778 & 2693 & 0.809892 \\
\hline 1.277555 & 0.293242 & 000170 & P01241 & 9049 & 2688 & 0.984313 \\
\hline 0.493149 & 1.077647 & 000170 & P10828 & 9049 & 7068 & 0.584497 \\
\hline 1.531827 & 0.038969 & 000170 & P10912 & 9049 & 2690 & 1.492859 \\
\hline 1.301202 & 0.269594 & 000170 & Q9UBX0 & 9049 & 8820 & 1.031608 \\
\hline 0.695151 & 0.875646 & 000170 & Q02643 & 9049 & 2692 & 0.180495 \\
\hline 1.44663 & 0.124166 & 000170 & P51692 & 9049 & 6777 & 1.322463 \\
\hline 1.369729 & 0.201067 & 000170 & Q9NWF9 & 9049 & 54476 & 1.168661 \\
\hline 1.463919 & 0.106877 & 000170 & P16473 & 9049 & 7253 & 1.357042 \\
\hline
\end{tabular}




\begin{tabular}{|c|c|c|c|c|c|c|}
\hline 0.841582 & 0.729215 & O00170 & Q969G2 & 9049 & 89884 & 0.112367 \\
\hline 0.743663 & 0.827133 & 000170 & P63092 & 9049 & 2778 & 0.08347 \\
\hline 0.967121 & 0.603675 & 000170 & Q9UBR4 & 9049 & 8022 & 0.363446 \\
\hline 1.133558 & 0.437238 & 000170 & Q01718 & 9049 & 4158 & 0.69632 \\
\hline 0.799039 & 0.771757 & 000170 & P41225 & 9049 & 6658 & 0.027282 \\
\hline 1.274641 & 0.296155 & 000170 & P07202 & 9049 & 7173 & 0.978486 \\
\hline 1.486163 & 0.084633 & 000170 & Q08499 & 9049 & 5144 & 1.40153 \\
\hline 1.161033 & 0.409763 & 000170 & P28069 & 9049 & 5449 & 0.751269 \\
\hline 0.897746 & 0.673051 & 000170 & P10644 & 9049 & 5573 & 0.224695 \\
\hline 0.969472 & 0.601324 & 000170 & Q06710 & 9049 & 7849 & 0.368148 \\
\hline 1.285706 & 0.28509 & 000170 & 075360 & 9049 & 5626 & 1.000616 \\
\hline 1.116321 & 0.454475 & 000170 & P32243 & 9049 & 5015 & 0.661847 \\
\hline 1.256809 & 0.313987 & 000170 & 095467 & 9049 & 2778 & 0.942821 \\
\hline 1.315857 & 0.254939 & 000170 & Q9NSE4 & 9049 & 55699 & 1.060919 \\
\hline 1.22072 & 0.350077 & 000170 & Q96T21 & 9049 & 79048 & 0.870643 \\
\hline 0.695137 & 0.875659 & 000170 & 060806 & 9049 & 9095 & 0.180522 \\
\hline 1.191189 & 0.379607 & 000170 & Q5JWF2 & 9049 & 2778 & 0.811582 \\
\hline 1.291872 & 0.278924 & 000170 & P01225 & 9049 & 2488 & 1.012948 \\
\hline 0.902885 & 0.667911 & 000170 & Q92847 & 9049 & 2693 & 0.234974 \\
\hline 1.264313 & 0.306483 & Q9NSE4 & P01241 & 55699 & 2688 & 0.95783 \\
\hline 1.287082 & 0.283715 & Q9NSE4 & Q06187 & 55699 & 695 & 1.003367 \\
\hline 1.295711 & 0.275086 & Q9NSE4 & P22888 & 55699 & 3973 & 1.020625 \\
\hline 1.239478 & 0.331318 & Q9NSE4 & P10828 & 55699 & 7068 & 0.90816 \\
\hline 1.315406 & 0.25539 & Q9NSE4 & P10912 & 55699 & 2690 & 1.060016 \\
\hline 1.4731 & 0.097696 & Q9NSE4 & Q96LT9 & 55699 & 55599 & 1.375404 \\
\hline 1.226141 & 0.344655 & Q9NSE4 & Q9UBX0 & 55699 & 8820 & 0.881485 \\
\hline 1.268621 & 0.302175 & Q9NSE4 & Q02643 & 55699 & 2692 & 0.966446 \\
\hline 1.335078 & 0.235718 & Q9NSE4 & P51692 & 55699 & 6777 & 1.09936 \\
\hline 1.268226 & 0.30257 & Q9NSE4 & Q9NWF9 & 55699 & 54476 & 0.965657 \\
\hline 1.298326 & 0.272471 & Q9NSE4 & P16473 & 55699 & 7253 & 1.025855 \\
\hline 1.213473 & 0.357323 & Q9NSE4 & Q969G2 & 55699 & 89884 & 0.85615 \\
\hline 1.409249 & 0.161547 & Q9NSE4 & P63092 & 55699 & 2778 & 1.247702 \\
\hline 1.272805 & 0.297992 & Q9NSE4 & Q9UBR4 & 55699 & 8022 & 0.974813 \\
\hline 1.224031 & 0.346765 & Q9NSE4 & Q01718 & 55699 & 4158 & 0.877265 \\
\hline 1.299074 & 0.271722 & Q9NSE4 & P41225 & 55699 & 6658 & 1.027352 \\
\hline 1.280999 & 0.289797 & Q9NSE4 & P07202 & 55699 & 7173 & 0.991202 \\
\hline 1.296319 & 0.274477 & Q9NSE4 & Q08499 & 55699 & 5144 & 1.021842 \\
\hline 1.214772 & 0.356025 & Q9NSE4 & P28069 & 55699 & 5449 & 0.858747 \\
\hline 1.08998 & 0.480816 & Q9NSE4 & P10644 & 55699 & 5573 & 0.609163 \\
\hline 1.155209 & 0.415587 & Q9NSE4 & Q06710 & 55699 & 7849 & 0.739622 \\
\hline 1.226141 & 0.344655 & Q9NSE4 & 075360 & 55699 & 5626 & 0.881485 \\
\hline
\end{tabular}




\begin{tabular}{|c|c|c|c|c|c|c|}
\hline 1.196441 & 0.374355 & Q9NSE4 & P32243 & 55699 & 5015 & 0.822087 \\
\hline 1.249051 & 0.321745 & Q9NSE4 & P84996 & 55699 & 2778 & 0.927306 \\
\hline 1.220246 & 0.350551 & Q9NSE4 & 095467 & 55699 & 2778 & 0.869695 \\
\hline 1.315857 & 0.254939 & Q9NSE4 & 000170 & 55699 & 9049 & 1.060919 \\
\hline 1.310034 & 0.260762 & Q9NSE4 & Q96T21 & 55699 & 79048 & 1.049272 \\
\hline 1.356183 & 0.214613 & Q9NSE4 & 060806 & 55699 & 9095 & 1.141569 \\
\hline 1.324088 & 0.246708 & Q9NSE4 & Q96P66 & 55699 & 83550 & 1.077381 \\
\hline 1.328342 & 0.242454 & Q9NSE4 & Q5JWF2 & 55699 & 2778 & 1.085888 \\
\hline 1.21652 & 0.354276 & Q9NSE4 & P01225 & 55699 & 2488 & 0.862244 \\
\hline 1.280219 & 0.290577 & Q9NSE4 & Q92847 & 55699 & 2693 & 0.989642 \\
\hline 1.196012 & 0.374785 & Q96T21 & P01241 & 79048 & 2688 & 0.821227 \\
\hline 1.325282 & 0.245515 & Q96T21 & Q06187 & 79048 & 695 & 1.079767 \\
\hline 1.422595 & 0.148202 & Q96T21 & P22888 & 79048 & 3973 & 1.274393 \\
\hline 1.339856 & 0.23094 & Q96T21 & P10828 & 79048 & 7068 & 1.108916 \\
\hline 1.318239 & 0.252557 & Q96T21 & P10912 & 79048 & 2690 & 1.065682 \\
\hline 1.332388 & 0.238409 & Q96T21 & Q96LT9 & 79048 & 55599 & 1.093979 \\
\hline 1.208142 & 0.362655 & Q96T21 & Q9UBX0 & 79048 & 8820 & 0.845487 \\
\hline 1.277836 & 0.29296 & Q96T21 & Q02643 & 79048 & 2692 & 0.984876 \\
\hline 1.287642 & 0.283155 & Q96T21 & P51692 & 79048 & 6777 & 1.004487 \\
\hline 1.386591 & 0.184205 & Q96T21 & Q9NWF9 & 79048 & 54476 & 1.202386 \\
\hline 1.274305 & 0.296492 & Q96T21 & P16473 & 79048 & 7253 & 0.977813 \\
\hline 1.453064 & 0.117732 & Q96T21 & Q969G2 & 79048 & 89884 & 1.335332 \\
\hline 1.427783 & 0.143014 & Q96T21 & P63092 & 79048 & 2778 & 1.284769 \\
\hline 1.409325 & 0.161471 & Q96T21 & Q9UBR4 & 79048 & 8022 & 1.247853 \\
\hline 1.23534 & 0.335457 & Q96T21 & Q01718 & 79048 & 4158 & 0.899883 \\
\hline 1.232987 & 0.33781 & Q96T21 & P41225 & 79048 & 6658 & 0.895177 \\
\hline 1.138106 & 0.43269 & Q96T21 & P07202 & 79048 & 7173 & 0.705416 \\
\hline 1.240415 & 0.330382 & Q96T21 & Q08499 & 79048 & 5144 & 0.910033 \\
\hline 1.349528 & 0.221268 & Q96T21 & P28069 & 79048 & 5449 & 1.128261 \\
\hline 1.314192 & 0.256604 & Q96T21 & P10644 & 79048 & 5573 & 1.057588 \\
\hline 1.195045 & 0.375752 & Q96T21 & Q06710 & 79048 & 7849 & 0.819293 \\
\hline 1.164328 & 0.406469 & Q96T21 & 075360 & 79048 & 5626 & 0.757859 \\
\hline 1.196647 & 0.37415 & Q96T21 & P32243 & 79048 & 5015 & 0.822497 \\
\hline 1.320837 & 0.24996 & Q96T21 & P84996 & 79048 & 2778 & 1.070877 \\
\hline 1.208142 & 0.362655 & Q96T21 & 095467 & 79048 & 2778 & 0.845487 \\
\hline 1.22072 & 0.350077 & Q96T21 & 000170 & 79048 & 9049 & 0.870643 \\
\hline 1.310034 & 0.260762 & Q96T21 & Q9NSE4 & 79048 & 55699 & 1.049272 \\
\hline 1.247978 & 0.322818 & Q96T21 & 060806 & 79048 & 9095 & 0.92516 \\
\hline 1.344479 & 0.226318 & Q96T21 & Q96P66 & 79048 & 83550 & 1.118161 \\
\hline 1.282706 & 0.28809 & Q96T21 & Q5JWF2 & 79048 & 2778 & 0.994616 \\
\hline 1.196012 & 0.374785 & Q96T21 & P01225 & 79048 & 2488 & 0.821227 \\
\hline
\end{tabular}




\begin{tabular}{|c|c|c|c|c|c|c|}
\hline 1.468365 & 0.102431 & Q96T21 & Q92847 & 79048 & 2693 & 1.365934 \\
\hline 1.040637 & 0.530159 & 060806 & P01241 & 9095 & 2688 & 0.510478 \\
\hline 1.425578 & 0.145219 & 060806 & Q06187 & 9095 & 695 & 1.280359 \\
\hline 1.55498 & 0.015816 & 060806 & P22888 & 9095 & 3973 & 1.539164 \\
\hline 1.414154 & 0.156643 & 060806 & P10912 & 9095 & 2690 & 1.257511 \\
\hline 1.061803 & 0.508993 & 060806 & Q9UBX0 & 9095 & 8820 & 0.55281 \\
\hline 0.34239 & 1.228407 & 060806 & Q02643 & 9095 & 2692 & 0.886017 \\
\hline 1.518076 & 0.05272 & 060806 & P51692 & 9095 & 6777 & 1.465356 \\
\hline 1.442912 & 0.127884 & 060806 & Q9NWF9 & 9095 & 54476 & 1.315028 \\
\hline 1.383992 & 0.186804 & 060806 & P16473 & 9095 & 7253 & 1.197188 \\
\hline 0.72843 & 0.842366 & 060806 & Q969G2 & 9095 & 89884 & 0.113937 \\
\hline 0.245777 & 1.325019 & 060806 & P63092 & 9095 & 2778 & 1.079242 \\
\hline 0.520566 & 1.05023 & 060806 & Q9UBR4 & 9095 & 8022 & 0.529664 \\
\hline 1.029536 & 0.54126 & 060806 & Q01718 & 9095 & 4158 & 0.488276 \\
\hline 0.541078 & 1.029718 & 060806 & P41225 & 9095 & 6658 & 0.48864 \\
\hline 1.2119 & 0.358897 & 060806 & P07202 & 9095 & 7173 & 0.853003 \\
\hline 1.457692 & 0.113104 & 060806 & Q08499 & 9095 & 5144 & 1.344588 \\
\hline 0.848804 & 0.721992 & 060806 & P28069 & 9095 & 5449 & 0.126812 \\
\hline 0.457144 & 1.113652 & 060806 & P10644 & 9095 & 5573 & 0.656508 \\
\hline 0.62742 & 0.943376 & 060806 & Q06710 & 9095 & 7849 & 0.315956 \\
\hline 1.098794 & 0.472003 & 060806 & 075360 & 9095 & 5626 & 0.626791 \\
\hline 0.831478 & 0.739318 & 060806 & P32243 & 9095 & 5015 & 0.092159 \\
\hline 0.964666 & 0.60613 & 060806 & 095467 & 9095 & 2778 & 0.358537 \\
\hline 0.695137 & 0.875659 & 060806 & 000170 & 9095 & 9049 & 0.180522 \\
\hline 1.356183 & 0.214613 & 060806 & Q9NSE4 & 9095 & 55699 & 1.141569 \\
\hline 1.247978 & 0.322818 & 060806 & Q96T21 & 9095 & 79048 & 0.92516 \\
\hline 1.354808 & 0.215989 & 060806 & Q5JWF2 & 9095 & 2778 & 1.138819 \\
\hline 1.074137 & 0.496659 & 060806 & P01225 & 9095 & 2488 & 0.577478 \\
\hline 0.443621 & 1.127175 & 060806 & Q92847 & 9095 & 2693 & 0.683554 \\
\hline 0.262568 & 1.308228 & Q96P66 & P01241 & 83550 & 2688 & 1.045661 \\
\hline 1.524339 & 0.046458 & Q96P66 & Q06187 & 83550 & 695 & 1.477881 \\
\hline 1.427758 & 0.143039 & Q96P66 & P22888 & 83550 & 3973 & 1.284719 \\
\hline 1.449864 & 0.120933 & Q96P66 & P10912 & 83550 & 2690 & 1.328931 \\
\hline 0.20086 & 1.369936 & Q96P66 & Q9UBX0 & 83550 & 8820 & 1.169076 \\
\hline 1.427745 & 0.143052 & Q96P66 & P51692 & 83550 & 6777 & 1.284693 \\
\hline 1.362986 & 0.20781 & Q96P66 & Q9NWF9 & 83550 & 54476 & 1.155176 \\
\hline 1.315581 & 0.255216 & Q96P66 & P16473 & 83550 & 7253 & 1.060365 \\
\hline 0.018742 & 1.552055 & Q96P66 & Q01718 & 83550 & 4158 & 1.533313 \\
\hline 1.298415 & 0.272381 & Q96P66 & P07202 & 83550 & 7173 & 1.026033 \\
\hline 1.345676 & 0.22512 & Q96P66 & Q08499 & 83550 & 5144 & 1.120556 \\
\hline 0.17642 & 1.394376 & Q96P66 & 075360 & 83550 & 5626 & 1.217957 \\
\hline
\end{tabular}




\begin{tabular}{|c|c|c|c|c|c|c|}
\hline 1.111115 & 0.459681 & Q96P66 & P84996 & 83550 & 2778 & 0.651434 \\
\hline 0.333373 & 1.237423 & Q96P66 & 095467 & 83550 & 2778 & 0.904049 \\
\hline 1.324088 & 0.246708 & Q96P66 & Q9NSE4 & 83550 & 55699 & 1.077381 \\
\hline 1.344479 & 0.226318 & Q96P66 & Q96T21 & 83550 & 79048 & 1.118161 \\
\hline 1.399912 & 0.170885 & Q96P66 & Q5JWF2 & 83550 & 2778 & 1.229027 \\
\hline 0.230656 & 1.34014 & Q96P66 & P01225 & 83550 & 2488 & 1.109484 \\
\hline 1.267266 & 0.303531 & Q5JWF2 & P01241 & 2778 & 2688 & 0.963735 \\
\hline 1.272306 & 0.29849 & Q5JWF2 & Q06187 & 2778 & 695 & 0.973815 \\
\hline 1.340824 & 0.229973 & Q5JWF2 & P22888 & 2778 & 3973 & 1.110851 \\
\hline 1.32569 & 0.245106 & Q5JWF2 & P10828 & 2778 & 7068 & 1.080584 \\
\hline 1.211346 & 0.359451 & Q5JWF2 & P10912 & 2778 & 2690 & 0.851895 \\
\hline 1.45071 & 0.120086 & Q5JWF2 & Q96LT9 & 2778 & 55599 & 1.330624 \\
\hline 1.236102 & 0.334694 & Q5JWF2 & Q9UBX0 & 2778 & 8820 & 0.901408 \\
\hline 1.416236 & 0.15456 & Q5JWF2 & Q02643 & 2778 & 2692 & 1.261676 \\
\hline 1.370419 & 0.200378 & Q5JWF2 & P51692 & 2778 & 6777 & 1.170041 \\
\hline 1.322354 & 0.248443 & Q5JWF2 & Q9NWF9 & 2778 & 54476 & 1.073911 \\
\hline 1.330701 & 0.240096 & Q5JWF2 & P16473 & 2778 & 7253 & 1.090605 \\
\hline 1.29766 & 0.273136 & Q5JWF2 & Q969G2 & 2778 & 89884 & 1.024525 \\
\hline 1.309296 & 0.2615 & Q5JWF2 & P63092 & 2778 & 2778 & 1.047797 \\
\hline 1.245408 & 0.325388 & Q5JWF2 & Q9UBR4 & 2778 & 8022 & 0.92002 \\
\hline 1.229742 & 0.341054 & Q5JWF2 & Q01718 & 2778 & 4158 & 0.888688 \\
\hline 1.302927 & 0.267869 & Q5JWF2 & P41225 & 2778 & 6658 & 1.035058 \\
\hline 1.224617 & 0.346179 & Q5JWF2 & P07202 & 2778 & 7173 & 0.878438 \\
\hline 1.307771 & 0.263025 & Q5JWF2 & Q08499 & 2778 & 5144 & 1.044746 \\
\hline 1.215962 & 0.354835 & Q5JWF2 & P28069 & 2778 & 5449 & 0.861127 \\
\hline 1.181285 & 0.389511 & Q5JWF2 & P10644 & 2778 & 5573 & 0.791774 \\
\hline 1.294907 & 0.27589 & Q5JWF2 & Q06710 & 2778 & 7849 & 1.019017 \\
\hline 1.298541 & 0.272255 & Q5JWF2 & 075360 & 2778 & 5626 & 1.026286 \\
\hline 1.359069 & 0.211728 & Q5JWF2 & P32243 & 2778 & 5015 & 1.147341 \\
\hline 1.420672 & 0.150124 & Q5JWF2 & P84996 & 2778 & 2778 & 1.270548 \\
\hline 1.211925 & 0.358872 & Q5JWF2 & 095467 & 2778 & 2778 & 0.853053 \\
\hline 1.191189 & 0.379607 & Q5JWF2 & 000170 & 2778 & 9049 & 0.811582 \\
\hline 1.328342 & 0.242454 & Q5JWF2 & Q9NSE4 & 2778 & 55699 & 1.085888 \\
\hline 1.282706 & 0.28809 & Q5JWF2 & Q96T21 & 2778 & 79048 & 0.994616 \\
\hline 1.354808 & 0.215989 & Q5JWF2 & 060806 & 2778 & 9095 & 1.138819 \\
\hline 1.399912 & 0.170885 & Q5JWF2 & Q96P66 & 2778 & 83550 & 1.229027 \\
\hline 1.236102 & 0.334694 & Q5JWF2 & P01225 & 2778 & 2488 & 0.901408 \\
\hline 1.192786 & 0.37801 & Q5JWF2 & Q92847 & 2778 & 2693 & 0.814776 \\
\hline 1.41773 & 0.153066 & P01225 & Q06187 & 2488 & 695 & 1.264664 \\
\hline 1.34683 & 0.223966 & P01225 & P22888 & 2488 & 3973 & 1.122864 \\
\hline 0.980467 & 0.590329 & P01225 & P10828 & 2488 & 7068 & 0.390138 \\
\hline
\end{tabular}




\begin{tabular}{|c|c|c|c|c|c|c|}
\hline 1.399484 & 0.171312 & P01225 & P10912 & 2488 & 2690 & 1.228172 \\
\hline 0.270143 & 1.300653 & P01225 & Q96LT9 & 2488 & 55599 & 1.03051 \\
\hline 1.114238 & 0.456558 & P01225 & Q02643 & 2488 & 2692 & 0.657681 \\
\hline 1.407656 & 0.16314 & P01225 & P51692 & 2488 & 6777 & 1.244516 \\
\hline 1.288588 & 0.282209 & P01225 & Q9NWF9 & 2488 & 54476 & 1.006379 \\
\hline 1.433063 & 0.137734 & P01225 & P16473 & 2488 & 7253 & 1.295329 \\
\hline 1.146281 & 0.424516 & P01225 & Q969G2 & 2488 & 89884 & 0.721765 \\
\hline 1.063493 & 0.507303 & P01225 & P63092 & 2488 & 2778 & 0.55619 \\
\hline 1.320472 & 0.250324 & P01225 & Q9UBR4 & 2488 & 8022 & 1.070149 \\
\hline 1.524662 & 0.046135 & P01225 & Q01718 & 2488 & 4158 & 1.478527 \\
\hline 1.160764 & 0.410032 & P01225 & P41225 & 2488 & 6658 & 0.750732 \\
\hline 1.332645 & 0.238151 & P01225 & P07202 & 2488 & 7173 & 1.094494 \\
\hline 1.299307 & 0.271489 & P01225 & Q08499 & 2488 & 5144 & 1.027818 \\
\hline 1.514932 & 0.055864 & P01225 & P28069 & 2488 & 5449 & 1.459068 \\
\hline 1.249079 & 0.321718 & P01225 & P10644 & 2488 & 5573 & 0.927361 \\
\hline 1.351299 & 0.219497 & P01225 & Q06710 & 2488 & 7849 & 1.131802 \\
\hline 1.291872 & 0.278924 & P01225 & 000170 & 2488 & 9049 & 1.012948 \\
\hline 1.21652 & 0.354276 & P01225 & Q9NSE4 & 2488 & 55699 & 0.862244 \\
\hline 1.196012 & 0.374785 & P01225 & Q96T21 & 2488 & 79048 & 0.821227 \\
\hline 1.074137 & 0.496659 & P01225 & 060806 & 2488 & 9095 & 0.577478 \\
\hline 0.230656 & 1.34014 & P01225 & Q96P66 & 2488 & 83550 & 1.109484 \\
\hline 1.236102 & 0.334694 & P01225 & Q5JWF2 & 2488 & 2778 & 0.901408 \\
\hline 1.231388 & 0.339408 & P01225 & Q92847 & 2488 & 2693 & 0.891981 \\
\hline 1.184614 & 0.386182 & Q92847 & P01241 & 2693 & 2688 & 0.798433 \\
\hline 1.405704 & 0.165092 & Q92847 & Q06187 & 2693 & 695 & 1.240612 \\
\hline 1.40101 & 0.169786 & Q92847 & P22888 & 2693 & 3973 & 1.231224 \\
\hline 0.325755 & 1.245042 & Q92847 & P10828 & 2693 & 7068 & 0.919287 \\
\hline 1.429716 & 0.141081 & Q92847 & P10912 & 2693 & 2690 & 1.288635 \\
\hline 1.241191 & 0.329606 & Q92847 & Q9UBX0 & 2693 & 8820 & 0.911585 \\
\hline 0.619764 & 0.951032 & Q92847 & Q02643 & 2693 & 2692 & 0.331268 \\
\hline 1.264745 & 0.306052 & Q92847 & P51692 & 2693 & 6777 & 0.958693 \\
\hline 1.29724 & 0.273556 & Q92847 & Q9NWF9 & 2693 & 54476 & 1.023684 \\
\hline 1.483427 & 0.08737 & Q92847 & P16473 & 2693 & 7253 & 1.396057 \\
\hline 0.778954 & 0.791843 & Q92847 & Q969G2 & 2693 & 89884 & 0.012889 \\
\hline 0.78674 & 0.784057 & Q92847 & P63092 & 2693 & 2778 & 0.002683 \\
\hline 0.823594 & 0.747202 & Q92847 & Q9UBR4 & 2693 & 8022 & 0.076391 \\
\hline 1.079356 & 0.49144 & Q92847 & Q01718 & 2693 & 4158 & 0.587916 \\
\hline 0.617378 & 0.953418 & Q92847 & P41225 & 2693 & 6658 & 0.336041 \\
\hline 1.295971 & 0.274825 & Q92847 & P07202 & 2693 & 7173 & 1.021146 \\
\hline 1.321346 & 0.24945 & Q92847 & Q08499 & 2693 & 5144 & 1.071896 \\
\hline 1.066575 & 0.504222 & Q92847 & P28069 & 2693 & 5449 & 0.562353 \\
\hline
\end{tabular}


bioRxiv preprint doi: https://doi.org/10.1101/2020.03.05.979781; this version posted March 6, 2020. The copyright holder for this preprint (which was not certified by peer review) is the author/funder. All rights reserved. No reuse allowed without permission.

\begin{tabular}{|l|c|c|c|c|c|c|}
0.884027 & 0.686769 & Q92847 & P10644 & 2693 & 5573 & 0.197259 \\
\hline 0.556829 & 1.013968 & Q92847 & Q06710 & 2693 & 7849 & 0.457139 \\
\hline 1.172249 & 0.398547 & Q92847 & O75360 & 2693 & 5626 & 0.773702 \\
\hline 1.026431 & 0.544365 & Q92847 & P32243 & 2693 & 5015 & 0.482067 \\
\hline 0.905209 & 0.665587 & Q92847 & P84996 & 2693 & 2778 & 0.239622 \\
\hline 1.190344 & 0.380452 & Q92847 & O95467 & 2693 & 2778 & 0.809892 \\
\hline 0.902885 & 0.667911 & Q92847 & O00170 & 2693 & 9049 & 0.234974 \\
\hline 1.280219 & 0.290577 & Q92847 & Q9NSE4 & 2693 & 55699 & 0.989642 \\
\hline 1.468365 & 0.102431 & Q92847 & Q96T21 & 2693 & 79048 & 1.365934 \\
\hline 0.443621 & 1.127175 & Q92847 & O60806 & 2693 & 9095 & 0.683554 \\
\hline 1.192786 & 0.37801 & Q92847 & Q5JWF2 & 2693 & 2778 & 0.814776 \\
\hline 1.231388 & 0.339408 & Q92847 & P01225 & 2693 & 2488 & 0.891981 \\
\hline
\end{tabular}

